Supporting Information

\title{
Probing Ligand Effects on the Ultrafast Dynamics of Copper Complexes via Midinfrared Pump-Probe and 2DIR Spectroscopies
}

Wei Weng, Alexander B. Weberg, Rahul Gera, Neil C. Tomson, Jessica M. Anna*

Department of Chemistry, University of Pennsylvania, Philadelphia, Pennsylvania 19104, United States

Table of Contents

S1 Synthetic procedures and structural characterizations $\quad$ S2

$\begin{array}{ll}\text { S1.1 General considerations } & \text { S2 }\end{array}$

$\mathrm{S} 1.2$ General procedure for the synthesis of $\left[{ }^{\mathrm{R} 3} \mathrm{P}_{3}\right.$ tren-Cu-N $\left.{ }_{3}\right] \mathrm{Bar}^{\mathrm{F}}{ }_{4}\left(\mathbf{1}^{\mathrm{PR} 3}\right) \quad \mathrm{S} 2$

S1.3 X-ray crystallographic details $\quad$ S3

S1.4 Evan's method measurements on $\mathbf{1}^{\text {PR3 }}$ S4

S1.5 UV-Vis spectra for $\mathbf{1}^{\text {PR3 }} \quad$ S6

S2 DFT-optimized coordinates and vibrational analysis $\quad$ S10

$\begin{array}{ll}\text { S2.1 Optimized geometry for } \mathbf{1}^{\text {PMe3 }} & \text { S10 }\end{array}$

$\begin{array}{ll}\text { S2.2 Optimized geometry for } \mathbf{1}^{\text {PMe2Ph }} & \text { S11 }\end{array}$

$\begin{array}{ll}\text { S2.3 Optimized geometry for } \mathbf{1}^{\text {PMePh2 }} & \text { S14 }\end{array}$

$\begin{array}{ll}\text { S2.4 Vibrational analysis results } & \text { S16 }\end{array}$

S3 Pump-probe spectra of $\mathbf{1}^{\text {PMe2Ph }}, \mathbf{1}^{\text {PMePh2 }}$, and free $\mathrm{N}_{3}^{-} \quad$ S18

S4 Justification for fitting functions of the anisotropy decay $\quad$ S19

S5 Estimation of $D_{m}^{D S E}$ based on the Debye-Stokes-Einstein equation $\quad$ S21

S6 Examining the reliability of $\mathrm{t}_{\mathrm{or}, 2}$ extracted from biexponential fitting $\quad$ S22

S7 Use of weighting functions when fitting anisotropy decay $\quad$ S24

S8 Detailed procedure to extract FFCF parameters $\quad$ S25

$\begin{array}{ll}\text { S9 Details of error propagation } & \text { S27 }\end{array}$

S9.1 Error propagation for the wobbling-in-a-cone analysis parameters $\quad$ S27

$\begin{array}{ll}\text { S9.2 Error propagation for the FFCF parameters } & \text { S27 }\end{array}$

$\begin{array}{lll}\text { S10 References } & \text { S29 }\end{array}$ 


\section{S1. Synthetic procedures and structural characterizations}

\section{S1.1 General considerations}

The synthetic procedures are based on previous work. ${ }^{1}$ All experiments were carried out under an atmosphere of purified nitrogen using standard dry, oxygen free glovebox techniques. All glassware, molecular sieves, stir bars, and Celite were dried in a $150{ }^{\circ} \mathrm{C}$ oven for a minimum of $12 \mathrm{~h}$ prior to use. Solvents (tetrahydrofuran, acetonitrile, diethyl ether, pentane) were dried by passage through a column of activated alumina and stored over $4 \AA$ molecular sieves under an inert atmosphere of dinitrogen. Deuterated acetonitrile was purchased from Cambridge Isotope Laboratory, dried over $\mathrm{CaH}_{2}$ prior to distillation, and stored over $3 \AA$ molecular sieves. All other reagents were obtained from commercial sources and used without further purification. ${ }^{1} \mathrm{H}$ NMR spectra for Evan's method measurements were recorded on a Bruker UNI 400 spectrometer.

\section{$\mathrm{S} 1.2$ General procedure for the synthesis of $\left[{ }^{\mathrm{R} 3} \mathrm{P}_{3}\right.$ tren-Cu-N $\left.\mathrm{N}_{3}\right] \mathrm{Bar}^{\mathrm{F}}{ }_{4}\left(\mathbf{1}^{\mathrm{PR} 3}\right)$}

The ${ }^{\mathrm{R} 3} \mathrm{P}_{3}$ tren ligand $(0.22 \mathrm{mmol}, 1.1$ equiv) was dissolved in $\mathrm{MeCN}(5 \mathrm{~mL})$, and the resulting solution was pipetted into a MeCN slurry $(3 \mathrm{~mL})$ of $\mathrm{CuCl}_{2}(27 \mathrm{mg}, 0.20 \mathrm{mmol}, 1$ equiv). The reaction mixture was stirred for $30 \mathrm{~min}$ at room temperature, at which point a green solution had formed. The reaction mixture was filtered through Celite to give a clear, green filtrate, to which a MeCN solution $(5 \mathrm{~mL})$ of $\operatorname{AgBAr}_{4}{ }_{4} \cdot 4 \mathrm{MeCN}$ (171 mg, $0.18 \mathrm{mmol}, 0.9$ equiv) was added. A white solid $(\mathrm{AgCl})$ was observed to precipitate immediately after addition. The reaction mixture was stirred in the dark for $10 \mathrm{~min}$, at which point the reaction mixture was filtered through Celite. Volatile materials were removed in vacuo to yield a green solid, which was taken up in diethyl ether $(5 \mathrm{~mL})$. The resulting slurry was filtered through Celite to remove a small amount of green solid and yield a clear green filtrate. Volatile materials were removed in vacuo, and the resulting solid was dissolved in THF ( $5 \mathrm{~mL}$ ). Sodium azide (13 mg, $0.2 \mathrm{mmol}, 1$ equiv) was added to the green THF solution, and the resulting slurry was stirred at room temperature for $2 \mathrm{~h}$, becoming more vibrantly green with time. The reaction mixture was filtered through Celite to remove insoluble materials $\left(\mathrm{NaCl}\right.$ and $\left.\mathrm{NaN}_{3}\right)$, and all volatile materials were removed in vacuo to yield $\mathbf{1}^{\mathrm{PR} 3}$ as a green solid. The solid was washed with pentane $(3 \times 5 \mathrm{~mL})$ and dried under vacuum for a minimum of $30 \mathrm{~min}$ prior to use.

1 $^{\text {PMe3: }}$ 54\% yield. Crystals of X-ray quality were grown from diethyl ether. Anal. Calcd. for $\mathrm{C}_{39} \mathrm{H}_{39} \mathrm{BCuF}_{20} \mathrm{~N}_{7} \mathrm{P}_{3}$ : C, 40.63; H, 3.41; N, 8.50. Found: $\mathrm{C}, 40.16 ; \mathrm{H}, 3.32 ; \mathrm{N}, 8.48$. IR (THF): $v_{\mathrm{NN}}$ $=2045 \mathrm{~cm}^{-1}$. UV-vis (THF) $\lambda_{\max }=389 \mathrm{~nm}\left(\varepsilon=2503 \mathrm{M}^{-1} \mathrm{~cm}^{-1}\right), \lambda_{\max }=740 \mathrm{~nm}, \lambda_{\max }=980 \mathrm{~nm}$. Evans's Method $\left(\mathrm{CD}_{3} \mathrm{CN}, 300 \mathrm{~K}\right): \mu_{\mathrm{eff}}=2.22 \mu_{\mathrm{B}} .{ }^{1} \mathrm{H}$ NMR $\left(400 \mathrm{MHz}, \mathrm{CD}_{3} \mathrm{CN}, 300 \mathrm{~K}\right): \delta=3.52$ (broad singlet) ppm.

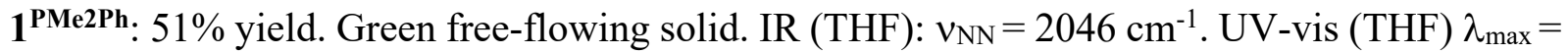
$394 \mathrm{~nm}\left(\varepsilon=2508 \mathrm{M}^{-1} \mathrm{~cm}^{-1}\right), \lambda_{\max }=732 \mathrm{~nm}, \lambda_{\max }=990 \mathrm{~nm}$. Evans's Method $\left(\mathrm{CD}_{3} \mathrm{CN}, 300 \mathrm{~K}\right): \mu_{\mathrm{eff}}$ $=2.25 \mu_{\mathrm{B}} .{ }^{1} \mathrm{H} \mathrm{NMR}\left(400 \mathrm{MHz}, \mathrm{CD}_{3} \mathrm{CN}, 300 \mathrm{~K}\right): \delta=9.66$ (broad singlet), $\delta=8.44$ (broad singlet), $\delta=7.34$ (broad singlet), $\delta=3.31$ (broad singlet) $\mathrm{ppm}$.

$1^{\text {PMePh2: }}$ 54\% yield. Green free-flowing solid. IR (THF): $v_{\mathrm{NN}}=2047 \mathrm{~cm}^{-1}$. UV-vis (THF) $\lambda_{\max }=$ $408 \mathrm{~nm}\left(\varepsilon=2276 \mathrm{M}^{-1} \mathrm{~cm}^{-1}\right), \lambda_{\max }=749 \mathrm{~nm}, \lambda_{\max }=961 \mathrm{~nm}$. Evans's Method $\left(\mathrm{CD}_{3} \mathrm{CN}, 300 \mathrm{~K}\right): \mu_{\mathrm{eff}}$ 
$=2.33 \mu_{\mathrm{B}} .{ }^{1} \mathrm{H} \mathrm{NMR}\left(400 \mathrm{MHz}, \mathrm{CD}_{3} \mathrm{CN}, 300 \mathrm{~K}\right): \delta=9.34$ (broad singlet), $\delta=8.55$ (broad singlet), $\delta=7.19$ (broad singlet) ppm.

S1.3 X-ray crystallographic details

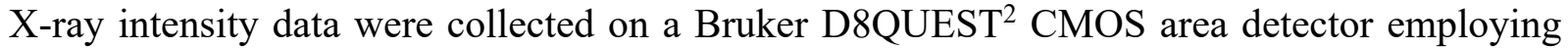
graphite-monochromated Mo-K $\alpha$ radiation $(\lambda=0.71073 \AA)$ at a temperature of $100 \mathrm{~K}$. Rotation frames were integrated using SAINT, ${ }^{3}$ producing a listing of unaveraged $\mathrm{F}^{2}$ and $\sigma\left(\mathrm{F}^{2}\right)$ values. The intensity data were corrected for Lorentz and polarization effects and for absorption using SADABS. ${ }^{4}$ The structure was solved by direct methods - ShelXT. ${ }^{5}$ Refinement was done by fullmatrix least squares based on $\mathrm{F}^{2}$ using SHELXL-2017. ${ }^{6}$ All reflections were used during refinement. The weighting scheme used was $\mathrm{w}=1 /\left[\sigma^{2}\left(\mathrm{~F}_{\mathrm{o}}^{2}\right)+(0.0369 \mathrm{P})^{2}+9.9284 \mathrm{P}\right]$ where $\mathrm{P}=$ $\left(\mathrm{F}_{\mathrm{o}}^{2}+2 \mathrm{~F}_{\mathrm{c}}^{2}\right) / 3$. Non-hydrogen atoms were refined anisotropically and hydrogen atoms were refined using a riding model.

Empirical formula
Formula weight
Temperature
Crystal system
Space group
a
b
c
$\beta$
Volume
$Z$
$d_{\text {calc }}$
$\mu$
F(000)
Crystal size, mm
$2 \theta$ range for data collection
Index ranges
Reflections collected
Independent reflections
Data/restraints/parameters
Goodness-of-fit on $F^{2}$
Final $R$ indexes [l>=2 $\sigma(\mathrm{I})]$
Final $R$ indexes [all data]
Largest diff. peak/hole

$\mathrm{C}_{43} \mathrm{H}_{49} \mathrm{BCuF}_{20} \mathrm{~N}_{7} \mathrm{OP}_{3}$
$1227.15 \mathrm{~g} / \mathrm{mol}$
$100 \mathrm{~K}$
monoclinic
$\mathrm{P} 2_{1} / \mathrm{c}$
$16.5506(10) \AA$
$20.5218(13) \AA$
$15.8428(10) \AA$
$105.663(3)^{\circ}$
$5181.2(6) \AA^{3}$
4
$1.573 \mathrm{~g} / \mathrm{cm}^{3}$
$0.629 \mathrm{~mm}^{-1}$
2492.0

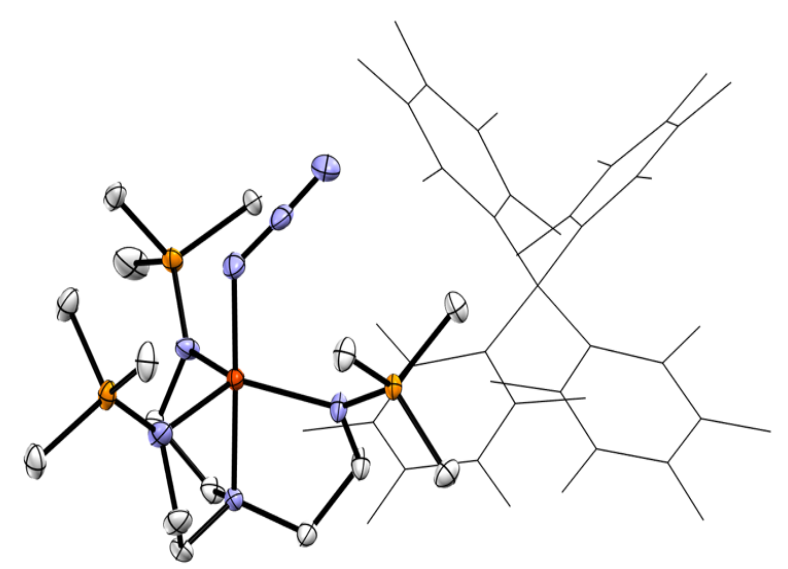

Figure S1. Molecular structure of $\mathbf{1}^{\mathrm{PMe}}$. The hydrogen atoms are removed for clarity. The thermal ellipsoids are shown at the $50 \%$ probability level.

$0.43 \times 0.25 \times 0.23$

$5.754-55.21^{\circ}$

$-21 \leq \mathrm{h} \leq 21,-26 \leq \mathrm{k} \leq 26,-20 \leq \mathrm{l} \leq 20$

129735

$11995[\mathrm{R}$ (int) $=0.0550]$

11995/0/696

1.051

$\mathrm{R}_{1}=0.0318, \mathrm{wR}_{2}=0.0722$

$\mathrm{R}_{1}=0.0410, \mathrm{wR}_{2}=0.0771$

$0.43 /-0.58$ e $\AA^{-3}$ 
S1.4 Evans's method measurements on $\mathbf{1}^{\text {PR3 }}$

Trimethoxybenzene (TMB) was both used as an internal standard in the solutions of $\mathbf{1}^{\text {PR3 }}$ and sealed as a $\mathrm{CD}_{3} \mathrm{CN}$ solution in a glass capillary tube as an external standard. ${ }^{1} \mathrm{H}$ NMR spectra were recorded on a Bruker UNI 400 spectrometer.

Table S1. Evan's method data for $\mathbf{1}^{\text {PR3 }}$.

\begin{tabular}{|lccc|}
\hline & $\mathbf{1}^{\text {PMe3 }}$ & $\mathbf{1}^{\text {PMe2Ph }}$ & $\mathbf{1}^{\text {PMePh2 }}$ \\
$\boldsymbol{\chi}_{\mathrm{D}}$ & $-5.41 \mathrm{E}-04$ & $-6.53 \mathrm{E}-04$ & $-7.65 \mathrm{E}-04$ \\
mass $(\mathbf{m g})$ & 13.2 & 8 & 11.9 \\
$\mathrm{MW}(\mathrm{g} / \mathrm{mol})$ & 1146 & 1338 & 1524 \\
vol. $(\mathbf{m L})$ & 0.65 & 0.6 & 0.6 \\
conc. $(\mathrm{M})$ & 0.018 & 0.010 & 0.013 \\
$\Delta$ ppm & 0.11 & 0.06 & 0.08 \\
$\Delta \mathrm{Hz}$ & 44 & 24 & 32 \\
Temp. $(\mathrm{K})$ & 300 & 300 & 300 \\
$\boldsymbol{\mu}_{\text {eff }}\left(\boldsymbol{\mu}_{\mathrm{B}}\right)$ & $2.22 \mathrm{muB}$ & $2.25 \mathrm{muB}$ & $\mathbf{2 . 3 3} \mathrm{mB}$ \\
\hline
\end{tabular}

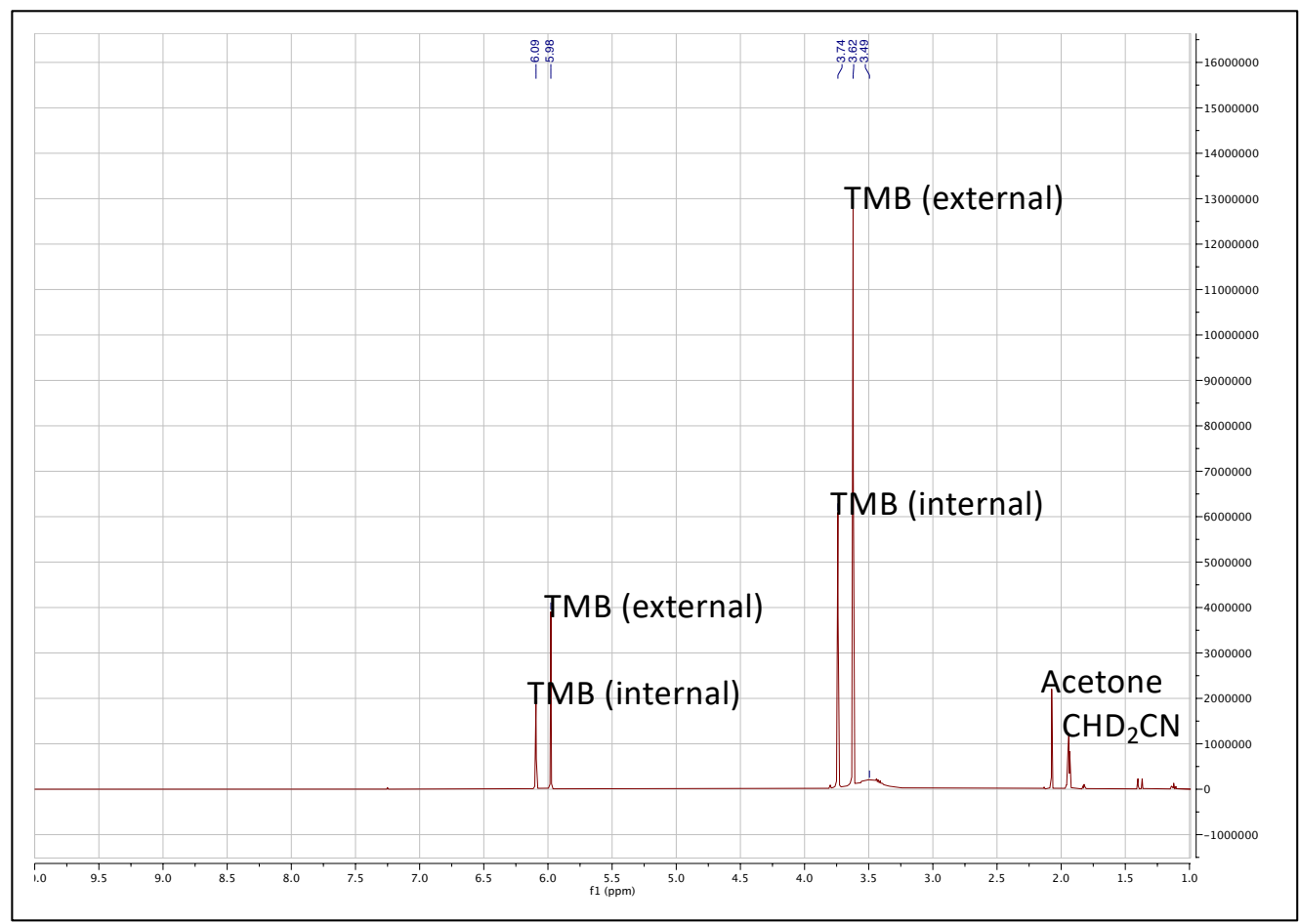

Figure S2. ${ }^{1} \mathrm{H}$ NMR spectrum of $\mathbf{1}^{\mathbf{P M e} 3}(400 \mathrm{MHz}, \mathrm{CD} 3 \mathrm{CN}, 300 \mathrm{~K}$ ) with both internal and external TMB standard. 


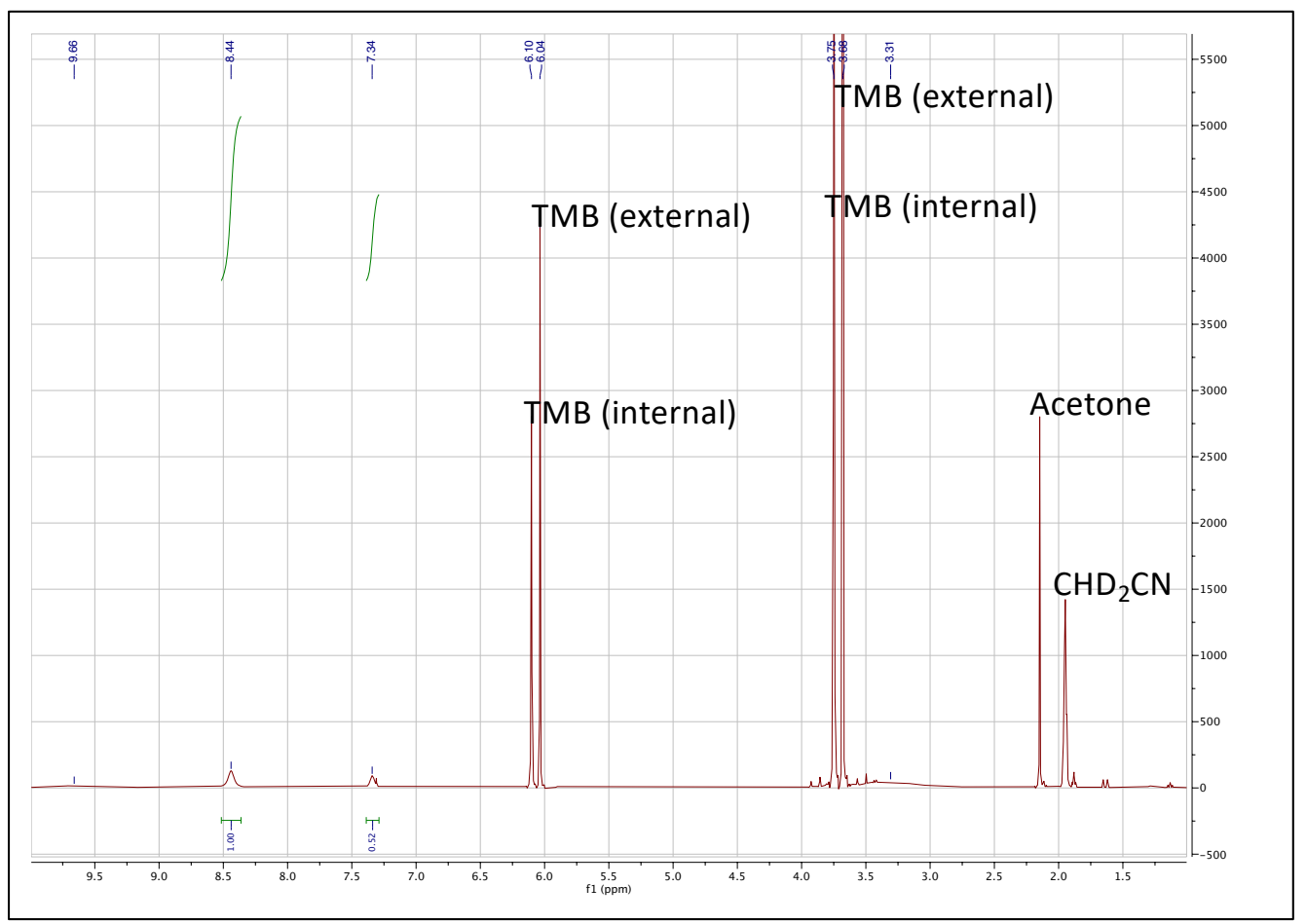

Figure S3. ${ }^{1} \mathrm{H}$ NMR spectrum of $\mathbf{1}^{\text {PMe2Ph }}\left(400 \mathrm{MHz}, \mathrm{CD}_{3} \mathrm{CN}, 300 \mathrm{~K}\right)$ with both internal and external TMB standard.

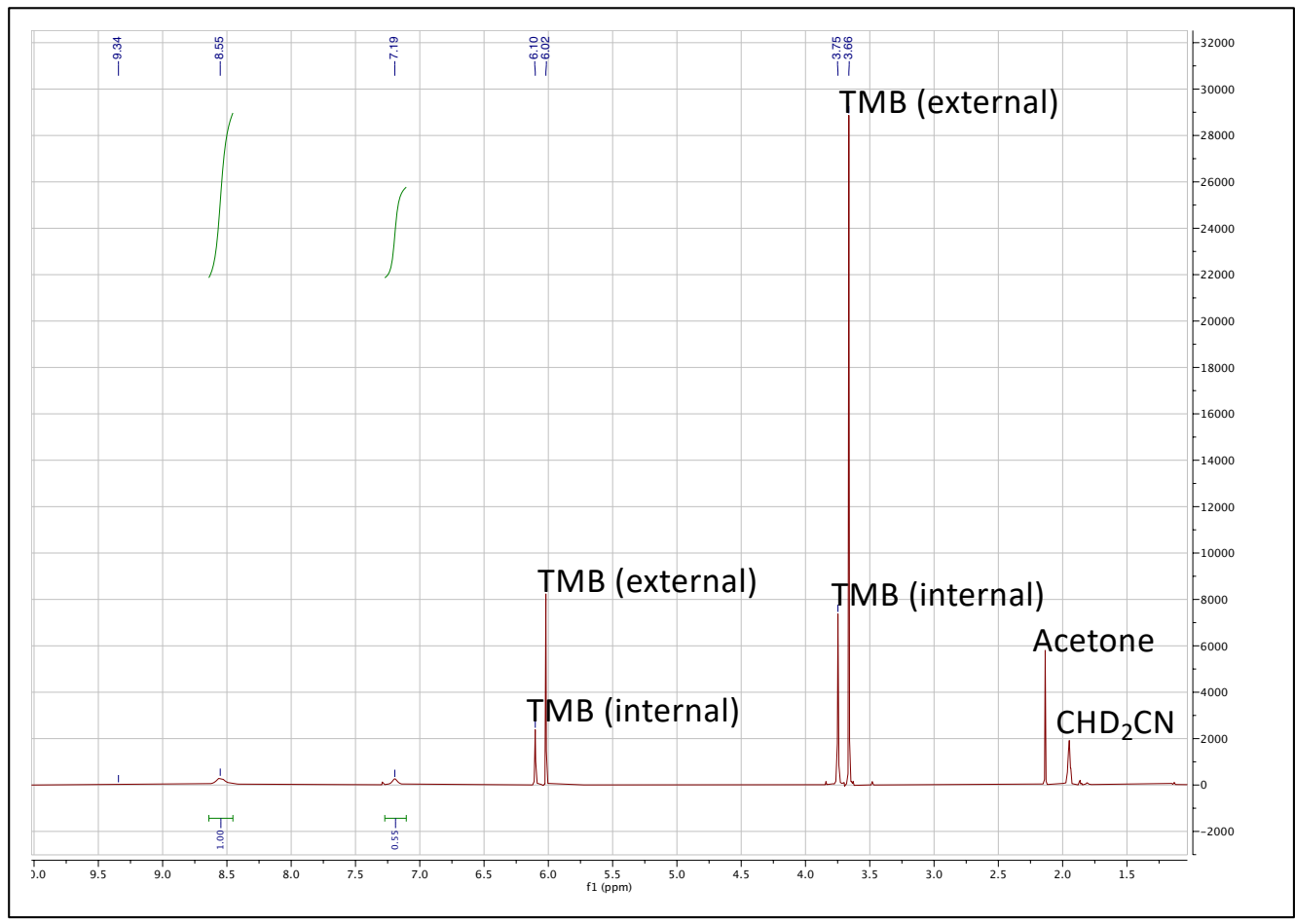

Figure S4. ${ }^{1} \mathrm{H}$ NMR spectrum of $\mathbf{1}^{\text {PMePh2 }}\left(400 \mathrm{MHz}, \mathrm{CD}_{3} \mathrm{CN}, 300 \mathrm{~K}\right)$ with both internal and external TMB standard. 
S1.5 UV-Vis spectra for $\mathbf{1}^{\mathbf{P R} 3}$

All UV-Vis spectra were recorded on a Cary 60 spectrophotometer using a quartz cuvette $(1 \mathrm{~cm}$ pathlength). All spectra were recorded on THF solutions of the analytes at $298 \mathrm{~K}$.

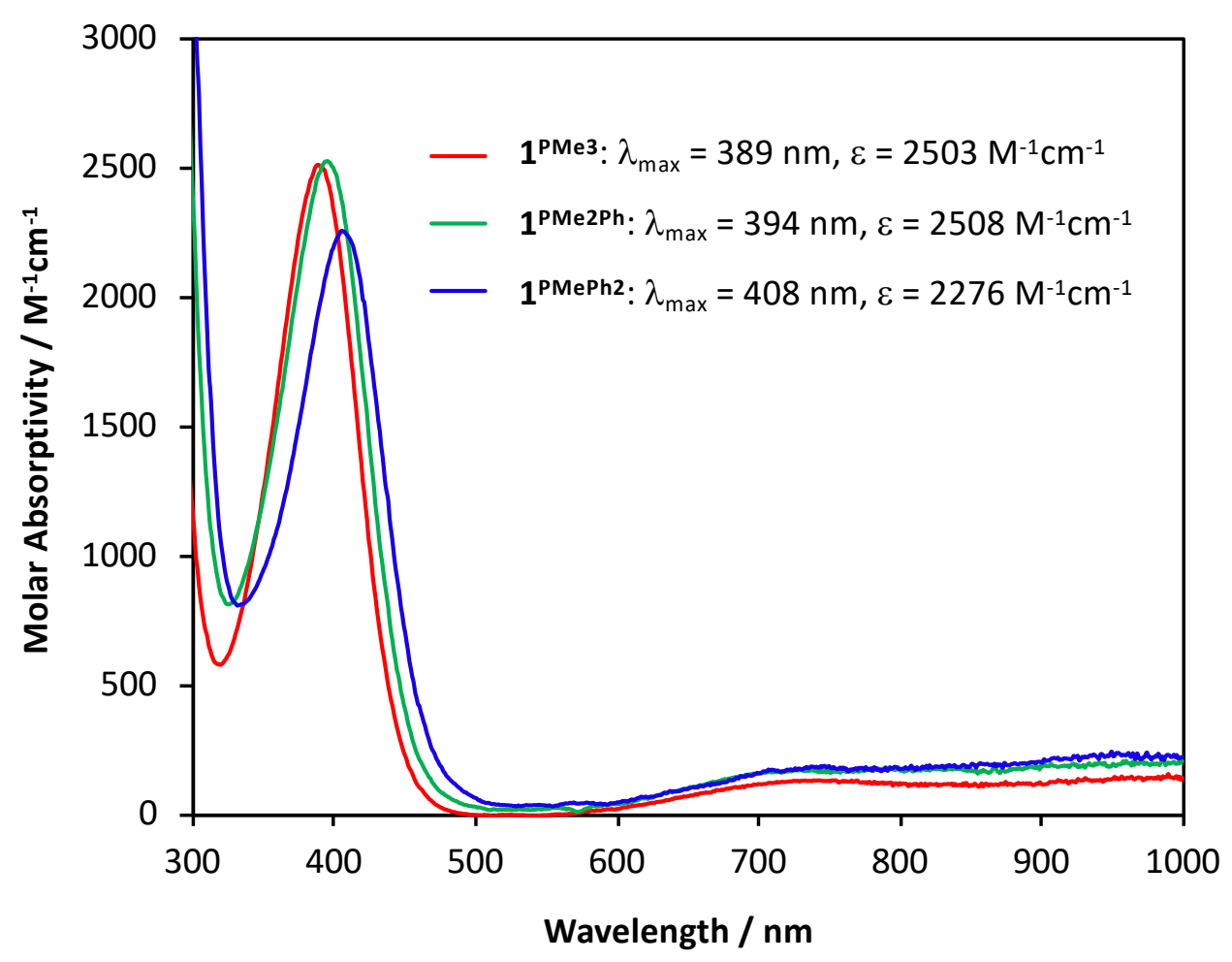

Figure S5. UV-Vis spectra of $\mathbf{1}^{\mathbf{P R 3}}$ in THF. 




Figure S6. UV-Vis spectra of $\mathbf{1}^{\mathrm{PMe} 3}$ at varying concentrations in THF.

\section{Molar Absorptivity of $\mathbf{3 8 9} \mathrm{nm}$ Feature for $\mathbf{1}^{\mathrm{PMe}}$}

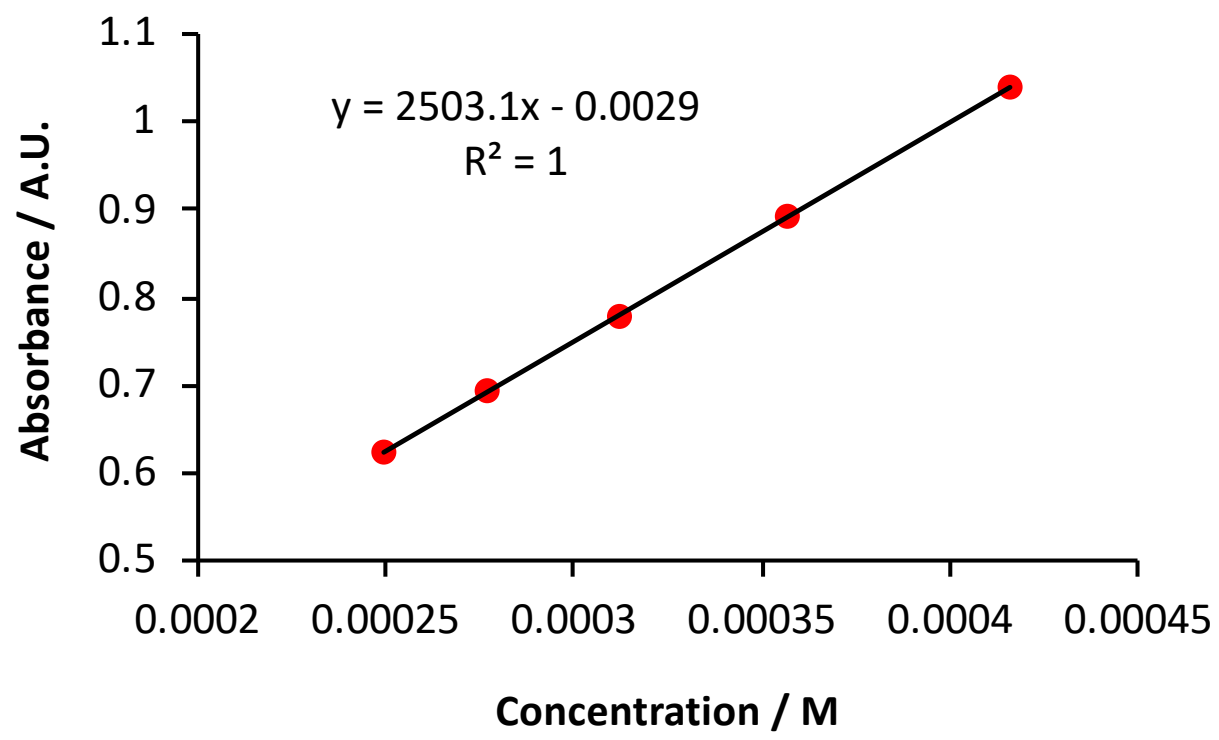

Figure S7. Plot of concentration vs. absorbance at $389 \mathrm{~nm}$, used to determine the molar absorptivity of $\lambda_{\max }$ for $1^{\text {PMe3 }}$. 


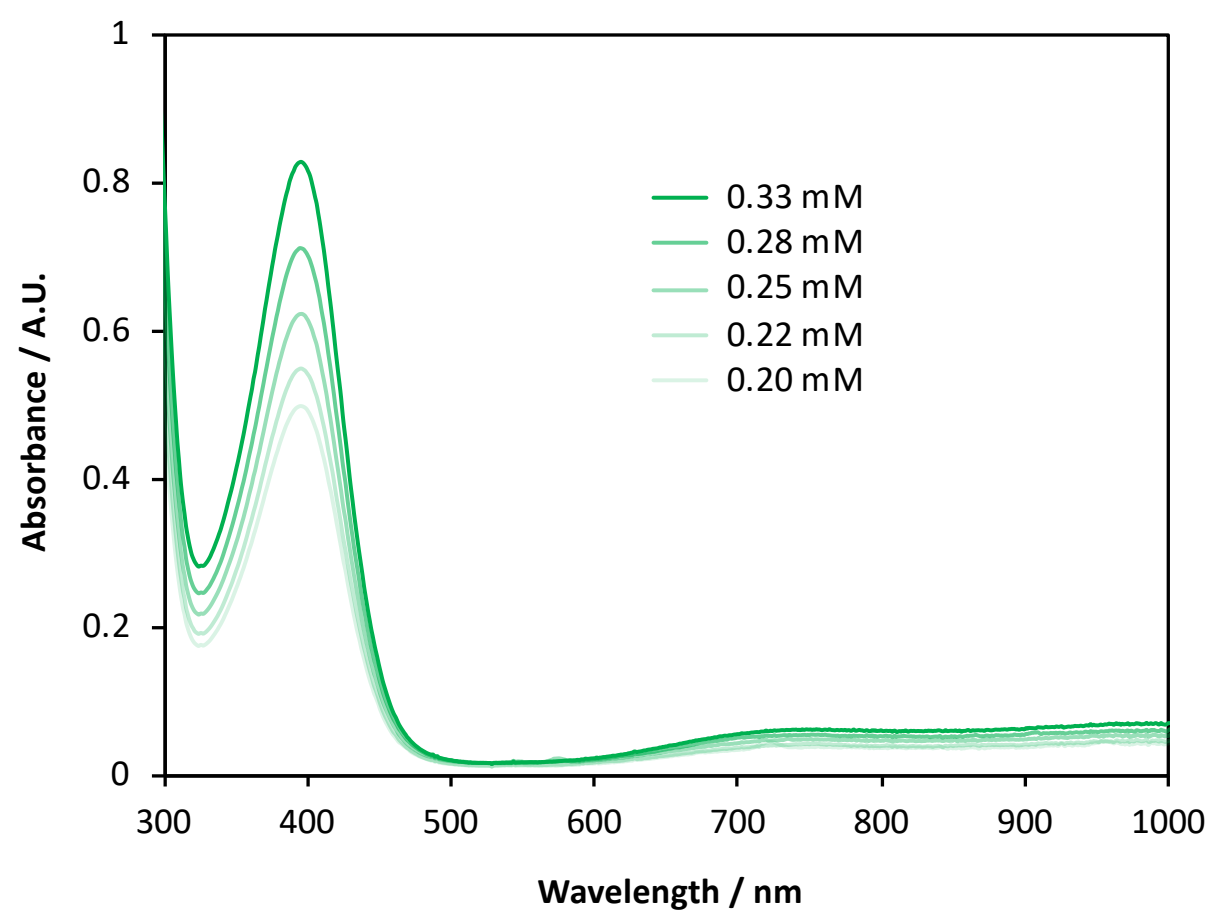

Figure S8. UV-Vis spectra of $\mathbf{1}^{\mathrm{PMe} 2 \mathrm{Ph}}$ at varying concentrations in THF.

\section{Molar Absorptivity of $394 \mathrm{~nm}$ Feature for $1^{\mathrm{PMe} 2 \mathrm{Ph}}$}

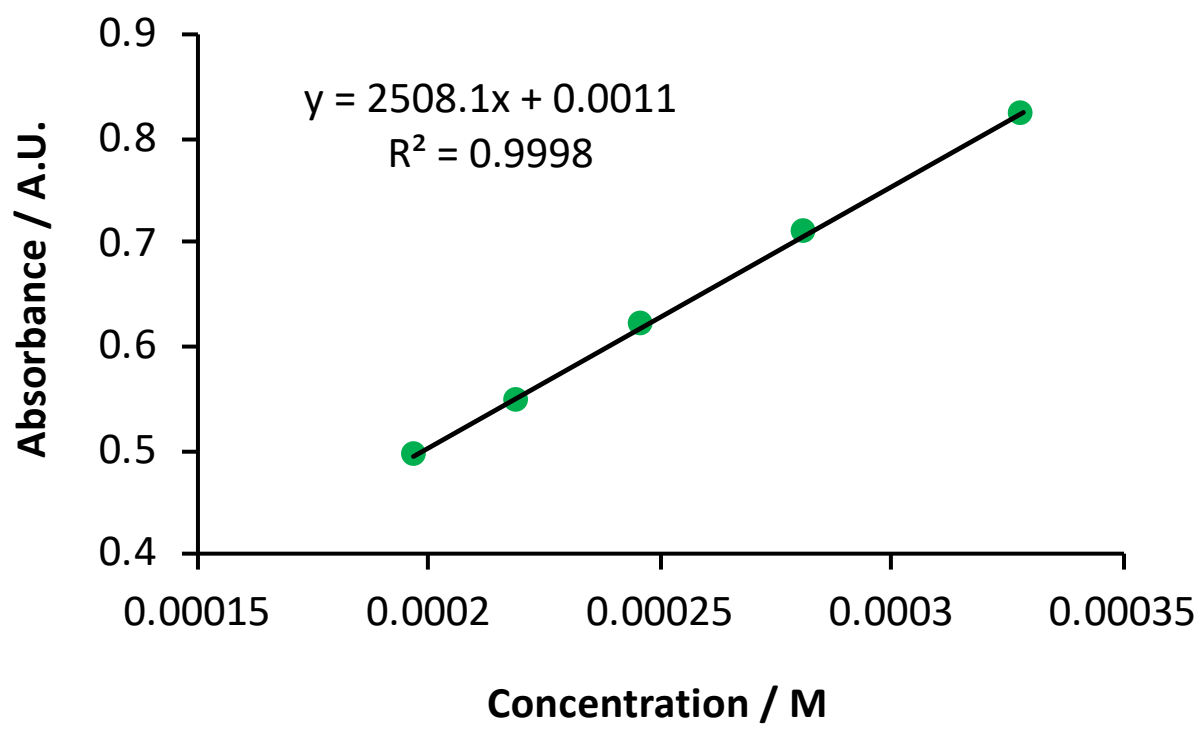

Figure S9. Plot of concentration vs. absorbance at $394 \mathrm{~nm}$, used to determine the molar absorptivity of $\lambda_{\max }$ for $\mathbf{1}^{\mathrm{PMe2Ph}}$. 


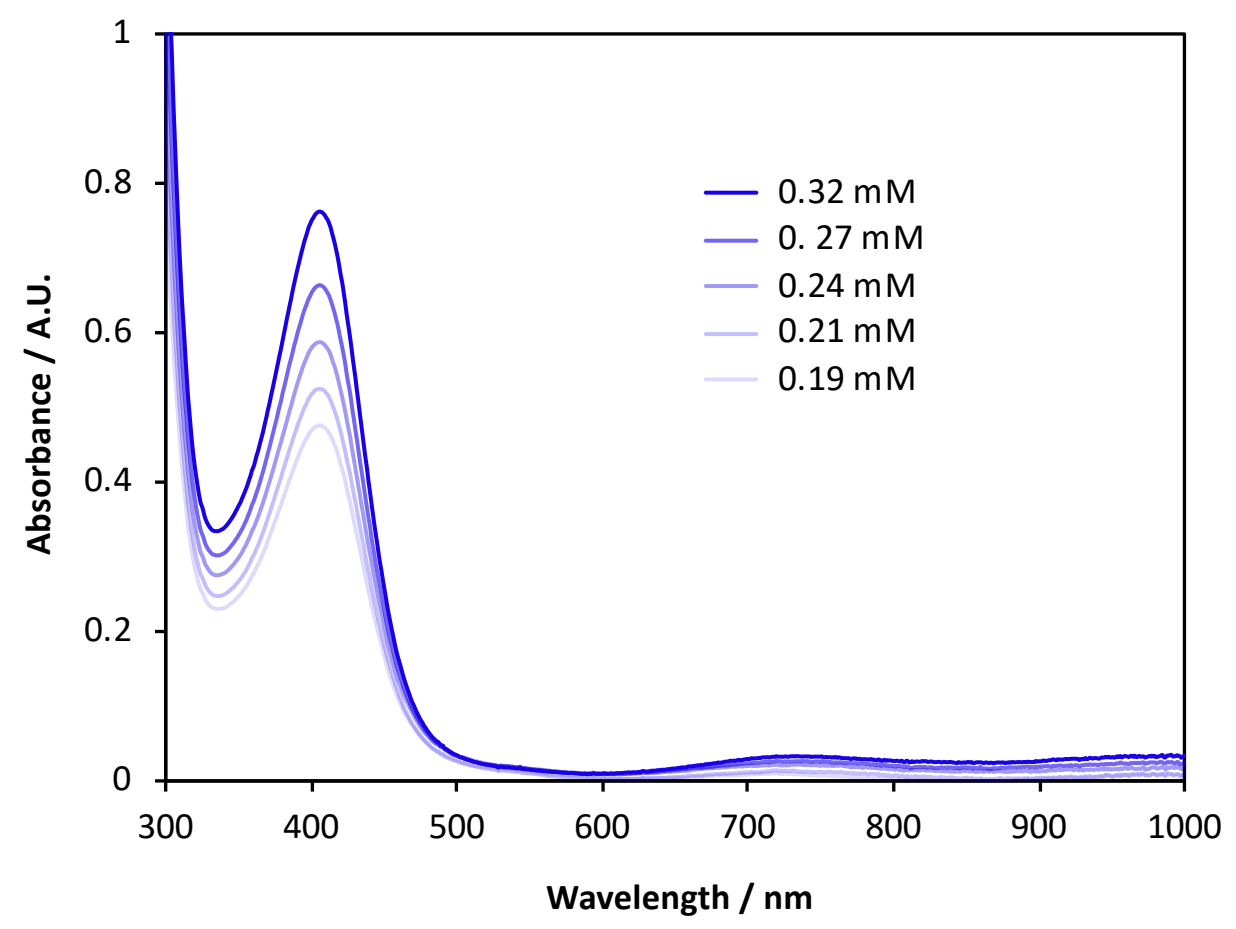

Figure S10. UV-Vis spectra of $\mathbf{1}^{\mathrm{PMePh} 2}$ at varying concentrations in THF.

Molar Absorptivity of $408 \mathrm{~nm}$ Feature for $\mathbf{1}^{\mathrm{PMePh} 2}$

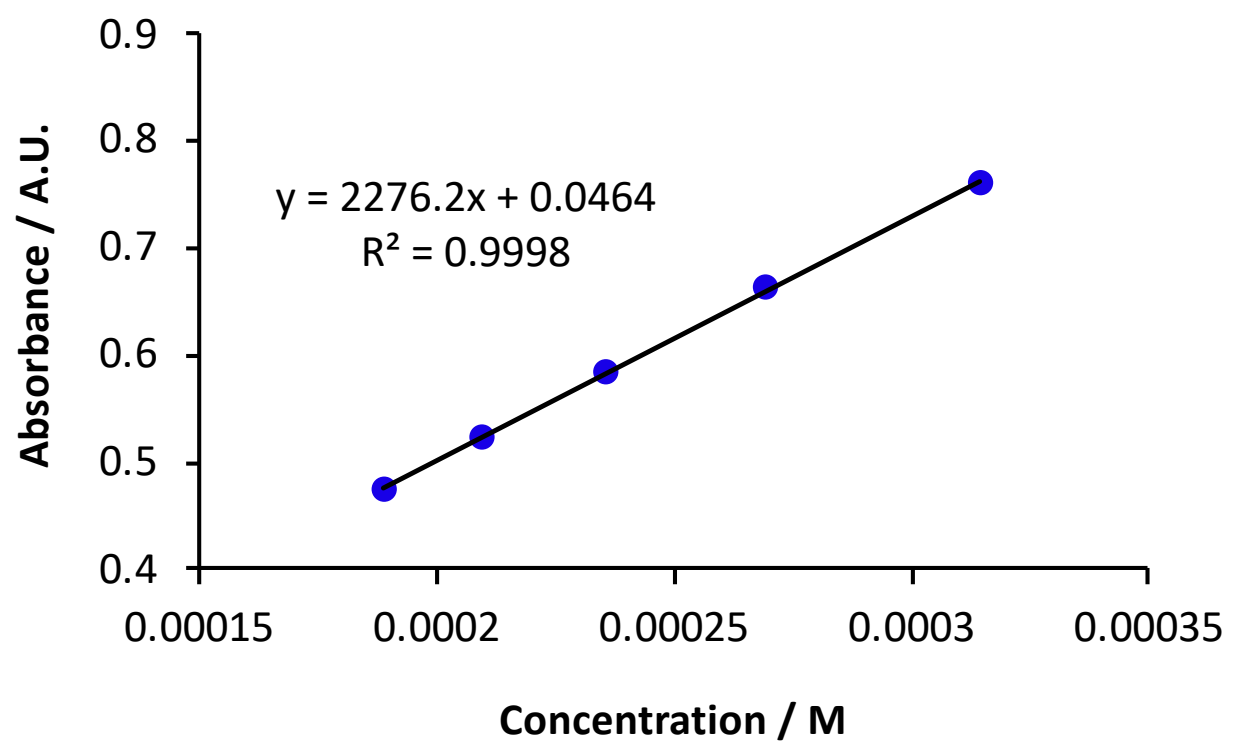

Figure S11. Plot of concentration vs. absorbance at $408 \mathrm{~nm}$, used to determine the molar absorptivity of $\lambda_{\max }$ for $1^{\text {PMePh2. }}$ 


\section{S2. DFT-optimized coordinates and vibrational analysis}

S2.1 Optimized geometry for $\mathbf{1}^{\text {PMe3 }}$

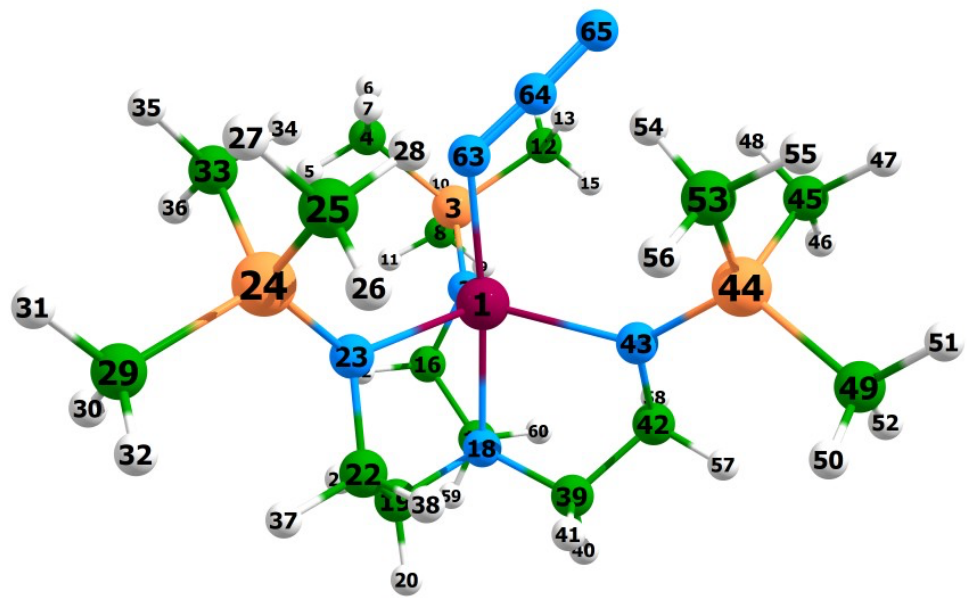

Figure S12. DFT-optimized structure for $\mathbf{1}^{\mathrm{PMe} 3}$.

Optimized cartesian coordinates (Angstroms):
Atom $\mathrm{X} \quad \mathrm{Y} \quad \mathrm{Z}$
$\mathrm{Cu} \quad 0.00000000 \quad 0.00000000 \quad 0.00000000$
$\mathrm{N}-1.99025400-0.75771700 \quad 0.37661800$
P $-3.11812100-1.20021000-0.69629800$
C $-3.58537700 \quad 0.06108000-1.94151400$
$\mathrm{H} \quad-4.00697700 \quad 0.93290700-1.43111200$
$\mathrm{H}-4.32404200-0.33577900-2.64533200$
$\mathrm{H} \quad-2.68599300 \quad 0.36683300-2.48196700$
C $-4.70139200-1.690559000 .08435600$
$\mathrm{H} \quad-4.52699900-2.47143500 \quad 0.83027800$
$\mathrm{H}-5.37538700-2.07925200-0.68485300$
$\mathrm{H} \quad-5.18074600-0.83413500 \quad 0.56643700$
C $-2.58177100-2.66743000-1.63248000$
$\mathrm{H}-1.65423900-2.48082900-2.17768700$
$\mathrm{H} \quad-3.35853900-2.94971600-2.35049000$
H $\quad-2.42355000-3.49148300-0.93072000$
C $-2.38991600-0.196769001 .67125900$
C $-1.25458900-0.457339002 .65325200$
N $\quad \begin{array}{llll}\text { C } & 0.02048400 & 0.12501300 & 2.15611200\end{array}$
C $\quad 0.13324900 \quad 1.56139700 \quad 2.52335900$
$\mathrm{H} \quad 0.51822400 \quad 1.654833003 .54921700$
$\mathrm{H} \quad-0.86952500 \quad 1.99478400 \quad 2.49580400$
C $\quad 1.00666400 \quad 2.31534100 \quad 1.52586900$
N $\quad 0.42823600 \quad 2.11553400 \quad 0.19672300$
P $\quad 0.701528003 .18038400-0.98865100$
C $2.158311002 .84696200-2.05185400$
H $3.065410002 .84165900-1.43917400$
$\mathrm{H} \quad 2.258686003 .60660800-2.83397700$
H $\quad 2.02944300 \quad 1.86398700-2.51207800$ 
C $\quad 0.948994004 .89176900-0.38143700$

$\mathrm{H} \quad 0.11383600 \quad 5.18487400 \quad 0.26153200$

$\mathrm{H} \quad 0.996085005 .57440000-1.23529100$

$\mathrm{H} \quad 1.88202900 \quad 4.97472000 \quad 0.18306400$

C $-0.722972003 .24676800-2.11707500$

$\mathrm{H} \quad-0.89624900 \quad 2.24375200-2.51307600$

$\mathrm{H} \quad-0.518963003 .94061400-2.93848200$

H $\quad-1.605709003 .58264500-1.56570900$

$\mathrm{H} \quad 1.022783003 .36982300 \quad 1.83783100$

$\mathrm{H} \quad 2.04642000 \quad 1.95581300 \quad 1.59135200$

C $\quad 1.18857700-0.65252700 \quad 2.64511900$

$\mathrm{H} \quad 1.02382800-0.951582003 .69070400$

H $\quad 2.06716500-0.003824002 .61294100$

C $\quad 1.45294000-1.860843001 .75220100$

N $\quad 1.68101500-1.37977300 \quad 0.38348300$

P $\quad 2.79677900-2.12271100-0.51835700$

C $\quad 2.28006100-3.67835400-1.34461600$

H $\quad 1.93749400-4.39323900-0.58970500$

H $\quad 3.11208000-4.12122800-1.90215900$

H $\quad 1.46065800-3.46060900-2.03579100$

C $\quad 4.30707500-2.58835500 \quad 0.41628700$

H $\quad 4.69910100-1.71384000 \quad 0.94390700$

H $\quad 5.06586700-2.95632200-0.28070000$

H $\quad 4.09458500-3.37846600 \quad 1.14141800$

C $3.37949500-1.02406600-1.84676600$

$\mathrm{H} \quad 2.58100500-0.82678000-2.56291900$

H $\quad 4.20802100-1.50684700-2.37394100$

H $\quad 3.72663900-0.08377400-1.41164000$

H $\quad 2.31249800-2.395437002 .18098300$

$\mathrm{H} \quad 0.60210000-2.55775100 \quad 1.80119800$

$\mathrm{H} \quad-1.48274900-0.056869003 .65141700$

$\mathrm{H}-1.12160400-1.538026002 .74332700$

H $\quad-3.29429000-0.665434002 .08413600$

$\mathrm{H} \quad-2.59626400 \quad 0.88431600 \quad 1.61520000$

$\mathrm{N} \quad-0.08755900 \quad 0.05778500-2.02543800$

$\mathrm{N} \quad 0.11316400-0.86703400-2.76510000$

$\mathrm{N} \quad 0.30115300-1.72962500-3.52779700$

Sum of electronic and thermal Free Energies $=-2197.922126$ Hartrees

S2.2 Optimized geometry for $\mathbf{1}^{\text {PMe2Ph }}$ 




Figure S13. DFT-optimized structure for $\mathbf{1}^{\mathrm{PMe} 2 \mathrm{Ph}}$.

Optimized cartesian coordinates (Angstroms):
Atom $\mathrm{X} \quad \mathrm{Y} \quad \mathrm{Z}$
$\mathrm{Cu} \quad 0.00000000 \quad 0.00000000 \quad 0.00000000$
$\mathrm{N} \quad 2.11075700-0.27724200-0.41324200$
P $\quad 3.34424700-0.38089800 \quad 0.62933500$
C $3.56278500 \quad 1.04864600 \quad 1.75469600$
H $\quad 3.73861000 \quad 1.96128100 \quad 1.17753800$
H $\quad 4.38718300 \quad 0.89372200 \quad 2.45717200$
H $\quad 2.62687400 \quad 1.162484002 .30878500$
C $3.13899700-1.829666001 .71485700$
H $\quad 2.22131400-1.767490002 .30229100$
H $\quad 3.99240400-1.890297002 .39725900$
H $\quad 3.10688700-2.73414100 \quad 1.10169200$
C $4.94593700-0.64335500-0.21378800$
C $\quad 5.10102300-1.78634500-1.01851800$
C $\quad 6.30449400-2.02541200-1.68070200$
C $\quad 7.36638000-1.12565700-1.54920300$
C $\quad 7.21994100 \quad 0.01376800-0.75652600$
C $\quad 6.01510500 \quad 0.25666200-0.09244600$
H $\quad 5.92017900 \quad 1.14823100 \quad 0.51845400$
H $\quad 8.04174000 \quad 0.71625500-0.65275900$
$\mathrm{H} \quad 8.30415700-1.31308700-2.06431500$
H $\quad 6.41384200-2.91266800-2.29753000$
H $\quad 4.27983000-2.48880200-1.13367200$
C $\quad 2.31255000 \quad 0.32977700-1.73414700$
C $1.28025600-0.28094800-2.67303700$
$\mathrm{N} \quad-0.09831600-0.04219000-2.16713900$
C $-0.61778100 \quad 1.27569500-2.62085700$
$\mathrm{H} \quad-1.06404900 \quad 1.17568000-3.62079200$
$\mathrm{H} \quad 0.22728200 \quad 1.96468400-2.69387700$
C $-1.610972001 .84596100-1.61445300$
$\mathrm{N} \quad-0.91792200 \quad 1.94210500-0.32777600$
P $\quad-1.38032900 \quad 3.02604300 \quad 0.77893900$
C $-2.70901100 \quad 2.48773600 \quad 1.92198700$
H $\quad-3.61224000 \quad 2.22254000 \quad 1.36450600$ 
H $\quad-2.94894900 \quad 3.25736800 \quad 2.66184000$

$\mathrm{H} \quad-2.33670400 \quad 1.59719300 \quad 2.43626600$

C $\quad 0.025531003 .43713200 \quad 1.85792000$

$\mathrm{H} \quad 0.38170700 \quad 2.51460600 \quad 2.32254400$

$\mathrm{H} \quad-0.29376800 \quad 4.14695500 \quad 2.62691400$

$\mathrm{H} \quad 0.82715600 \quad 3.88221200 \quad 1.26277200$

C $-1.93170700 \quad 4.59944900 \quad 0.02690800$

C $\quad-3.21940000 \quad 5.12009000 \quad 0.22395300$

C $-3.59235600 \quad 6.32478000-0.37710700$

C $-2.686053007 .01682100-1.18211000$

C $-1.402568006 .50275400-1.38926000$

C $-1.027278005 .30091700-0.79022800$

$\mathrm{H} \quad-0.02985400 \quad 4.90560900-0.96349400$

$\mathrm{H} \quad-0.69501600 \quad 7.03726000-2.01645000$

$\mathrm{H} \quad-2.977884007 .95356500-1.64823600$

$\mathrm{H} \quad-4.59104700 \quad 6.71975400-0.21508800$

$\mathrm{H} \quad-3.93779800 \quad 4.59662300 \quad 0.84625300$

$\mathrm{H} \quad-1.95147700 \quad 2.82045000-1.99234300$

$\mathrm{H} \quad-2.50276700 \quad 1.20064000-1.56141500$

C $-1.01250300-1.14856500-2.55486800$

$\mathrm{H} \quad-0.77124900-1.49075700-3.57181300$

H $\quad-2.03365100-0.76019900-2.56252600$

C $-0.94272200-2.29002300-1.54527500$

$\mathrm{N}-1.29359500-1.75118100-0.22196200$

P $\quad-2.28174800-2.62611200 \quad 0.70974100$

C $-1.51490700-4.045102001 .58291200$

H $-1.07003400-4.74048000 \quad 0.86465300$

H $\quad-2.23893200-4.57863900 \quad 2.20640900$

H $\quad-0.72547000-3.64148100 \quad 2.22443900$

C $-3.00321700-1.597758002 .02786000$

$\mathrm{H} \quad-2.24816300-1.351654002 .77637500$

$\mathrm{H} \quad-3.80398000-2.164336002 .51226300$

H $\quad-3.41816600-0.68325400 \quad 1.59861500$

C $-3.70491000-3.29781400-0.22919700$

C $-4.52133700-2.39099400-0.92825400$

C $-5.62215200-2.84552900-1.65312900$

C $-5.91656700-4.21174100-1.69462000$

C $-5.10741800-5.12005000-1.01024900$

C $-4.00569900-4.66695100-0.27999600$

$\mathrm{H} \quad-3.38782100-5.38792100 \quad 0.24506900$

$\mathrm{H} \quad-5.33032600-6.18255300-1.04305900$

$\mathrm{H} \quad-6.77346000-4.56590900-2.26052100$

H $\quad-6.24884700-2.13589000-2.18560200$

H $\quad-4.29298500-1.32855300-0.90973700$

$\mathrm{H} \quad-1.62575700-3.07897900-1.89149000$

$\mathrm{H} \quad 0.06574900-2.72970800-1.54143700$

H $\quad 1.37559500 \quad 0.11421800-3.69438300$

H $\quad 1.44700200-1.36027300-2.70969200$

$\mathrm{H} \quad 3.30958200 \quad 0.12781200-2.14897200$

$\mathrm{H} \quad 2.19670300 \quad 1.42508500-1.71160200$

$\mathrm{N} \quad \begin{array}{llll}0.11832700 & 0.21134300 & 2.01176800\end{array}$ 
$\begin{array}{llll}\mathrm{N} & 0.11727700 & -0.69324800 & 2.80157700\end{array}$

N $\quad 0.11838700-1.535109003 .60934900$

Sum of electronic and thermal Free Energies $=-2772.961155$ Hartrees

S2.3 Optimized geometry for $\mathbf{1}^{\text {PMePh2 }}$



Figure S14. DFT-optimized structure for $\mathbf{1}^{\text {PMePh2 }}$.

Optimized cartesian coordinates (Angstroms):
Atom $\mathrm{X} \quad \mathrm{Y} \quad \mathrm{Z}$
$\begin{array}{lllll}\mathrm{Cu} & 0.00000000 & 0.00000000 & 0.00000000\end{array}$
$\mathrm{N} \quad 2.11660700-0.17427600-0.41341400$
P $\quad 3.37084900-0.62009200 \quad 0.49633600$
C $\quad 2.94757400-2.05894600 \quad 1.53295600$
$\mathrm{H} \quad 2.17679500-1.806629002 .26290900$
H $\quad 3.83694500-2.407227002 .06552800$
H $\quad 2.57802800-2.86404700 \quad 0.89243400$
C $4.81015400-1.14448100-0.50879800$
C $\quad 4.74442900-2.36221500-1.20914200$
C $\quad 5.80793300-2.77105900-2.01317100$
C $\quad 6.94490200-1.96662000-2.13341300$
C $7.01467400-0.75200100-1.44898900$
C $\quad 5.95303700-0.34086200-0.63978600$
$\mathrm{H} \quad 6.01952200 \quad 0.60280100-0.10812200$
H $\quad 7.89519900-0.12283500-1.54120400$
$\mathrm{H} \quad 7.77296100-2.28680100-2.75930600$
H $\quad 5.74984300-3.71706300-2.54366400$
H $\quad 3.86543000-2.99554800-1.13037200$
C $\quad 3.99914400 \quad 0.66231400 \quad 1.64262900$
C $\quad 4.91736500 \quad 0.362755002 .66340800$ 
C $\quad \begin{array}{llll}5.37784700 & 1.37137600 & 3.50963500\end{array}$

C $\quad 4.930976002 .685706003 .34206700$

C 4.021083002 .990344002 .32794300

C $3.553759001 .98136900 \quad 1.48267900$

$\mathrm{H} \quad 2.83402400 \quad 2.20861000 \quad 0.70237700$

$\mathrm{H} \quad 3.67185400 \quad 4.010690002 .19712200$

H $\quad 5.292031003 .469292004 .00216600$

H $\quad 6.08482100 \quad 1.13197100 \quad 4.29868600$

H $\quad 5.28169800-0.651358002 .80053400$

C $\quad 2.30498900 \quad 0.36943000-1.76178300$

C $1.29108400-0.30467700-2.68056700$

$\mathrm{N}-0.09359900-0.09518100-2.17834300$

C $-0.65266200 \quad 1.18904700-2.67644200$

$\mathrm{H} \quad-1.11529500 \quad 1.03707800-3.66222700$

$\mathrm{H} \quad 0.17460800 \quad 1.89286600-2.79775600$

C $-1.641375001 .78463100-1.67984000$

$\mathrm{N} \quad-0.93294000 \quad 1.96215300-0.40815500$

P $-1.409610003 .11527800 \quad 0.61872600$

C $-0.09831300 \quad 3.40073400 \quad 1.84306600$

$\mathrm{H} \quad 0.16081700 \quad 2.45950400 \quad 2.33352800$

H $\quad-0.42080000 \quad 4.149060002 .57206000$

H $\quad 0.77419700 \quad 3.78194600 \quad 1.30633200$

C $-1.652103004 .73346600-0.21336400$

C $-2.62121600 \quad 5.66123100 \quad 0.19814100$

C $-2.70586500 \quad 6.91106400-0.41949500$

C $-1.827473007 .24471000-1.45243700$

C $\quad-0.85706100 \quad 6.32800800-1.86610200$

C $-0.767389005 .08024900-1.24897100$

$\mathrm{H} \quad-0.010362004 .37070600-1.57102500$

H $\quad-0.170809006 .58375000-2.66831000$

H $\quad-1.897363008 .21642800-1.93297900$

H $\quad-3.459619007 .62184900-0.09320200$

H $\quad-3.31055800 \quad 5.41421800 \quad 0.99887300$

C $\quad-2.96317800 \quad 2.78909100 \quad 1.53716800$

C $-4.18058500 \quad 2.73556900 \quad 0.83303000$

C $\quad-5.36593500 \quad 2.41008800 \quad 1.49322100$

C $\quad-5.35052600 \quad 2.13023500 \quad 2.86313100$

C $\quad-4.147115002 .175892003 .56917600$

C $\quad-2.95814300 \quad 2.50061700 \quad 2.91104500$

$\mathrm{H} \quad-2.03183400 \quad 2.524369003 .47525300$

$\mathrm{H} \quad-4.12991600 \quad 1.960622004 .63358500$

H $\quad-6.27410900 \quad 1.880067003 .37716400$

$\mathrm{H} \quad-6.30087900 \quad 2.38232100 \quad 0.94084800$

$\mathrm{H} \quad-4.20951300 \quad 2.96509000-0.22873600$

$\mathrm{H} \quad-2.01345300 \quad 2.72828700-2.10562700$

$\mathrm{H} \quad-2.51612600 \quad 1.12280300-1.57651300$

C $-0.97345800-1.24259600-2.52569300$

$\mathrm{H} \quad-0.73132900-1.60663100-3.53525800$

H $\quad-2.00750100-0.88976600-2.53433200$

C $-0.84861000-2.34737100-1.47907700$

$\mathrm{N}-1.18336300-1.79937900-0.15247900$ 


$$
\begin{array}{lrrr}
\mathrm{P} & -2.54956400 & -2.31921700 & 0.53981700 \\
\mathrm{C} & -2.92552500 & -1.33686200 & 2.02105800 \\
\mathrm{H} & -2.19612000 & -1.57840700 & 2.79784600 \\
\mathrm{H} & -3.92502300 & -1.59906300 & 2.37828000 \\
\mathrm{H} & -2.88013300 & -0.26882000 & 1.80144800 \\
\mathrm{C} & -4.02946800 & -2.24320700 & -0.54282200 \\
\mathrm{C} & -4.77529200 & -1.05331400 & -0.61495300 \\
\mathrm{C} & -5.84409800 & -0.94318700 & -1.50502500 \\
\mathrm{C} & -6.17523900 & -2.01330600 & -2.34073000 \\
\mathrm{C} & -5.43675000 & -3.19679800 & -2.28115600 \\
\mathrm{C} & -4.37071100 & -3.31345200 & -1.38673700 \\
\mathrm{H} & -3.81722500 & -4.24618100 & -1.33810500 \\
\mathrm{H} & -5.69181000 & -4.03296600 & -2.92576500 \\
\mathrm{H} & -7.00779000 & -1.92558400 & -3.03291100 \\
\mathrm{H} & -6.42006000 & -0.02292700 & -1.54296800 \\
\mathrm{H} & -4.53307200 & -0.21020800 & 0.02531300 \\
\mathrm{C} & -2.44607200 & -4.05827700 & 1.10942500 \\
\mathrm{C} & -3.58062500 & -4.77147000 & 1.53224800 \\
\mathrm{C} & -3.44828300 & -6.06978100 & 2.02494600 \\
\mathrm{C} & -2.18634400 & -6.66747200 & 2.09639800 \\
\mathrm{C} & -1.05510700 & -5.96356900 & 1.67896900 \\
\mathrm{C} & -1.18296700 & -4.66178700 & 1.19001100 \\
\mathrm{H} & -0.30556200 & -4.10584100 & 0.87383500 \\
\mathrm{H} & -0.07347600 & -6.42523100 & 1.73531400 \\
\mathrm{H} & -2.08676500 & -7.67977300 & 2.47781600 \\
\mathrm{H} & -4.32944500 & -6.61520700 & 2.35038300 \\
\mathrm{H} & -4.56796400 & -4.32208100 & 1.47311500 \\
\mathrm{H} & -1.48521800 & -3.18658600 & -1.79238000 \\
\mathrm{H} & 0.17859600 & -2.73593600 & -1.46225200 \\
\mathrm{H} & 1.37206500 & 0.06095300 & -3.71447500 \\
\mathrm{H} & 1.49459700 & -1.37838700 & -2.68539300 \\
\mathrm{H} & 3.30827100 & 0.18549500 & -2.16833700 \\
\mathrm{H} & 2.15618000 & 1.45981700 & -1.77558100 \\
\mathrm{~N} & 0.07690900 & 0.22838300 & 1.99456600 \\
\mathrm{~N} & 0.11626400 & -0.66121500 & 2.79951700 \\
\mathrm{~N} & 0.15083100 & -1.48368200 & 3.62556700
\end{array}
$$

Sum of electronic and thermal Free Energies $=-3347.995352$ Hartrees

\section{S2.4 Vibrational analysis results}

From the DFT calculations we find that the asymmetric stretching mode $\left(v_{\text {asym }}\right)$, symmetric stretching mode $\left(v_{\text {sym }}\right)$, and bending modes $\left(v_{\text {bend }}\right)$ of the copper bound azide group are mostly localized over the three nitrogen atoms of azide. The scaled vibrational frequencies for the four vibrational modes associated with azide are reported in Table S2. The scaling factors for the $\mathbf{1}^{\mathbf{P R} 3}$ series and free $\mathrm{N}_{3}^{-}$were determined by comparing the calculated frequencies to the asymmetric stretching mode of the experimental spectra. For the $\mathbf{1}^{\mathbf{P R 3}}$ series we found that a scaling factor of 0.953 minimized the difference between the scaled frequencies and experimental values. For free $\mathrm{N}_{3}^{-}$we found that a scaling factor of 0.931 gave the best agreement between the experimental and 
scaled frequencies. Note that different scaling factors were used to account for the different basis sets used for the copper complexes (LANL2DZ/6-31G(d)) and free $\mathrm{N}_{3}^{-}(6-31 \mathrm{G}(\mathrm{d})$ ).

Table S2. The scaled vibrational frequencies and scaling factors for the $\mathbf{1}^{\mathrm{PR} 3}$ series and free $\mathrm{N}_{3}^{-}$

\begin{tabular}{|c|c|c|c|c|}
\hline & Scaling factor & $v_{\text {asym }}\left(\mathrm{cm}^{-1}\right)$ & $v_{\text {sym }}\left(\mathrm{cm}^{-1}\right)$ & $v_{\text {bend }}\left(\mathrm{cm}^{-1}\right)$ \\
\hline \multirow{2}{*}{$\mathbf{1}^{\text {PMe3 }}$} & \multirow{2}{*}{0.953} & 2047 & 1322 & 631,592 \\
\cline { 3 - 5 } $\mathbf{1}^{\text {PMe2Ph }}$ & 2046 & 1322 & 630,592 \\
\cline { 3 - 5 } & & 2047 & 1326 & 631,593 \\
\hline $\mathbf{1}^{\text {PMePh2 }}$ & 1995 & 1276 & 614,614 \\
\hline Free N ${ }_{3}^{-}$ & 0.931 & \multicolumn{3}{|c}{} \\
\hline
\end{tabular}

From Table S2 we can see that the relative spacing between $v_{\text {asym }}$ and $v_{\text {sym }}$ among the four samples are similar, and that the values of $v_{\text {bend }}$ are comparable for the bound and free azide groups. The calculated frequencies show that the energy gap between $v_{\text {asym }}$ and the sum of $v_{\text {sym }}$ and $v_{\text {bend }}$ are similar for copper-bound azide and free $\mathrm{N}_{3}^{-}$, suggesting that the intramolecular vibrational energy redistribution (IVR) within the azide group proceeds with a similar time constant for bound and free azide $(\sim 2.3 \mathrm{ps})$. We note that this is only an estimate as the $v_{\text {bend }}+$ $v_{\text {sym }}$ combination band was obtained from the harmonic vibrational frequencies. The 2.3 ps time constant for IVR is comparable to the overall decay time constant extracted for copper bound azide $(\sim 4.5 \mathrm{ps})$. If the population relaxation process is dominated by the longer time scale component as in free $\mathrm{N}_{3}^{-}$, we would not be able to resolve a $\sim 2$ ps IVR component. Thus the IVR component associated with the copper bound azide may not be resolvable in our measurements. Future work will focus on comparing the results of an anharmonic vibrational analysis to further investigate the mechanism of IVR for the copper bound azide complexes. 


\section{S3. Pump-probe spectra of $1^{\mathrm{PMe} 2 \mathrm{Ph}}, 1^{\mathrm{PMePh} 2}$, and free $\mathrm{N}_{3}^{-}$}

The time-resolved pump-probe spectra of $\mathbf{1}^{\mathbf{P M e 2 P h}}, \mathbf{1}^{\mathbf{P M e P h} 2}$, and free $\mathrm{N}_{3}^{-}$are shown in Figure S15. Spectra displayed in the left and middle columns were taken under parallel and perpendicular polarization conditions respectively. The positive peak (yellow) arises from the $0 \rightarrow 1$ transition of the azide asymmetric stretch and the negative peak (blue) from the $1 \rightarrow 2$ transition. The dashed vertical lines superposed on the pump-probe spectra denote the frequencies where the time traces are taken, and the traces for the parallel $\left(\Delta \mathrm{I}_{\|}(\mathrm{t})\right)$ and perpendicular signal $\left(\Delta \mathrm{I}_{\perp}(\mathrm{t})\right)$ are displayed in the right column. The anisotropy and population relaxations presented in the main text were obtained from the traces.
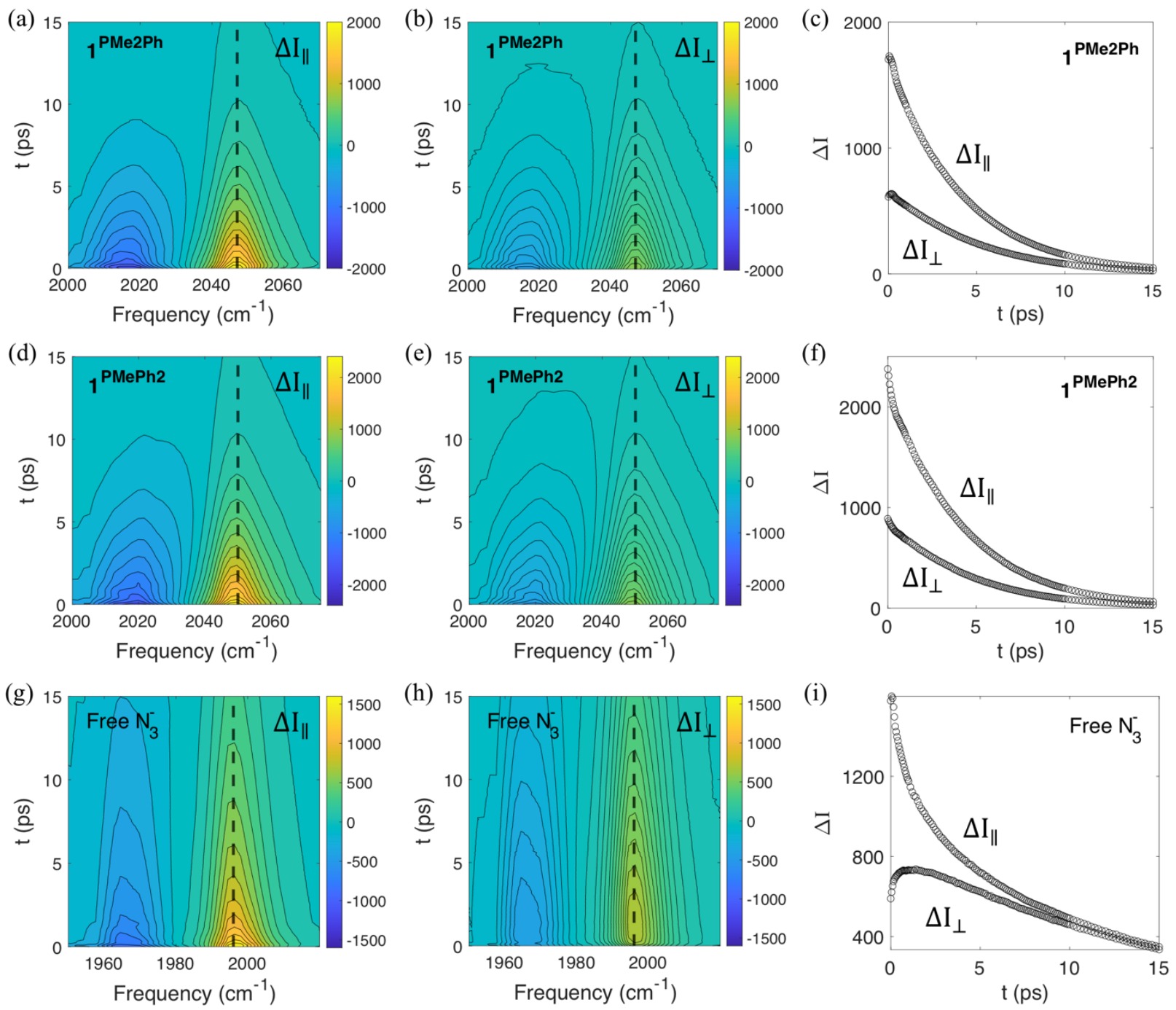

Figure S15. Experimental pump-probe spectra of $\mathbf{1}^{\mathrm{PMe2Ph}}, \mathbf{1}^{\mathrm{PMePh} 2}$, and free $\mathrm{N}_{3}^{-}$under parallel ((a), (d), and $\left.(\mathrm{g})\right)$ and perpendicular ((b), (e), and (h)) polarizations. The dashed vertical lines denote the frequencies at which the time traces are taken. The time traces of the parallel and perpendicular signal of each sample are shown on the right column ((c), (f), and (i)). 


\section{S4. Justification for fitting functions of the anisotropy decay}

In the main text the anisotropy decay curves of the copper-bound azide are fit with biexponential functions:

$$
r(t)=a_{1} \exp \left(-\frac{t}{t_{1, o r}}\right)+a_{2} \exp \left(-\frac{t}{t_{2, o r}}\right)
$$

However, the detection time window is limited by the vibrational lifetime of azide which is not long enough to characterize the complete orientational relaxation dynamics. This is especially evident for $\mathbf{1}^{\text {PMePh2}}$, where the slow time constant extracted from the biexponential fitting (58.6 ps) is over 12 times the vibrational lifetime $(4.6 \mathrm{ps})$. In fact, the anisotropy decay for the three copper complexes can also be fit with a monoexponential function plus a constant term:

$$
r(t)=a_{1} \exp \left(-\frac{t}{t_{1, o r}}\right)+a_{2}
$$

The fitting parameters obtained with both fitting functions (Eq. S1 and Eq. S2) are presented in Table S3.

Table S4 summarizes the wobbling-in-a-cone analysis parameters extracted from both fitting functions given by Equations $S 1$ and $S 2$. Here we report the total cone semiangle $\left(\theta_{\text {tot }}\right)$ and the correlation time associated with restricted diffusive motion $\left(\tau_{c}\right)$ for comparison purposes. In Equation S2, the constant term, a2, can be viewed as the amplitude of a non-decaying component.

Examining Table S3 and S4, we find that fitting with a monoexponential plus a constant term significantly increases the time constant and relative contribution of the fast decay component. Moreover, the difference in the anisotropy decay curves among the three copper complexes at later delay time is mostly manifested in the constant term. This leads to relatively large and more distinct $\theta_{\text {tot }}$ values. With biexponential fitting, the slow component has a major contribution to the anisotropy decay and the decay curve differences at later delay time are reflected by the slow time constants. As a result, the $\theta_{\text {tot }}$ values are smaller with less variation among the three copper complexes.

We take the biexponential fitting as a better description for the anisotropy decay for two reasons. First, when fit with a single exponential function plus a constant offset (Equation S2), $\theta_{\text {tot }}$ of $\mathbf{1}^{\text {PMe3 }}$ in Table S4 is the same as that of free $\mathrm{N}_{3}^{-}$, which is not expected as the free azide should experience less spatial restriction than the bound azide. Second, from Section S5 of the SI, we learn that the orientational diffusion coefficients of the copper complexes estimated from the Debye-StokesEinstein equation, $\mathrm{D}_{\mathrm{m}}^{\mathrm{DSE}}$, decrease with the addition of phenyl groups under both the slip and stick boundary conditions. The complete orientational relaxation of the bound azide group is closely related to the orientational diffusion of the entire copper complex. Thus we expect to see a similar decreasing trend for $\mathrm{D}_{\mathrm{m}}$, the experimentally measured complete diffusion coefficient for bound azide, which is consistent with an increasing trend of $\tau_{m}\left(t_{2, o r}\right)$. This increasing trend of $t_{2, \text { or }}$ can not be obtained by fitting with a single exponential. Based on these two reasons we use a biexponential function to fit the anisotropy decay curves. 
Table S3. Optimized parameters extracted from fitting the anisotropy decay of the three copper complexes with fitting functions given by Equations S1 and S2. Parameters of free $N_{3}^{-}$extracted from biexponential fitting are shown for comparison. The errors in this table represent $95 \%$ confidence interval.

\begin{tabular}{|c|c|c|c|c|c|}
\hline Samples & $\begin{array}{c}\text { Fitting } \\
\text { functions }\end{array}$ & $a_{1}$ & $\mathrm{t}_{1, \mathrm{or}}(\mathrm{ps})$ & $a_{2}$ & $\mathrm{t}_{2, \mathrm{or}}(\mathrm{ps})$ \\
\hline \multirow{2}{*}{$1^{\text {PMe3 }}$} & Eq. S2 & $0.23 \pm 0.01$ & $8.4 \pm 0.4$ & $0.11 \pm 0.01$ & - \\
\hline & Eq. S1 & $0.06 \pm 0.02$ & $3.1 \pm 0.8$ & $0.28 \pm 0.02$ & $21 \pm 2$ \\
\hline \multirow{2}{*}{$1^{\mathrm{PMe} 2 \mathrm{Ph}}$} & Eq. S2 & $0.16 \pm 0.01$ & $8.1 \pm 0.6$ & $0.19 \pm 0.01$ & - \\
\hline & Eq. S1 & $0.05 \pm 0.01$ & $2.5 \pm 0.7$ & $0.30 \pm 0.01$ & $37 \pm 5$ \\
\hline \multirow{2}{*}{$1^{\mathrm{PMePh} 2}$} & Eq. S2 & $0.12 \pm 0.01$ & $9.0 \pm 0.8$ & $0.23 \pm 0.01$ & - \\
\hline & Eq. S1 & $0.02 \pm 0.01$ & $2.7 \pm 1.8$ & $0.32 \pm 0.01$ & $59 \pm 12$ \\
\hline Free $\mathrm{N}_{3}^{-}$ & Eq. S1 & $0.18 \pm 0.01$ & $1.2 \pm 0.1$ & $0.10 \pm 0.01$ & $6.4 \pm 0.4$ \\
\hline
\end{tabular}

Table S4. The $\theta_{\text {tot }}$ and $\tau_{c}$ parameters from the wobbling-in-a-cone analysis for the three copper complexes extracted from the optimized parameters from the fitting functions given by Equations $\mathrm{S} 1$ and $\mathrm{S} 2 . \theta_{\text {tot }}$ and $\tau_{c}$ parameters of free $N_{3}^{-}$were extracted from a biexponential fit and are shown for comparison. The errors in this table are propagated from those in Table S3 and the details of error propagation can be found in Section S9.1 of the SI.

\begin{tabular}{|c|c|c|c|}
\hline Samples & Fitting functions & $\theta_{\text {tot }}\left({ }^{\circ}\right)$ & $\tau_{\mathrm{c}}(\mathrm{ps})$ \\
\hline \multirow{2}{*}{$\mathbf{1}^{\mathbf{P M e} 3}$} & Eq. S2 & $51 \pm 1$ & $8.4 \pm 0.4$ \\
\cline { 2 - 4 } & Eq. S1 & $27 \pm 2$ & $3.7 \pm 1.1$ \\
\hline \multirow{2}{*}{$\mathbf{1}^{\text {PMe2Ph }}$} & Eq. S2 & $39 \pm 1$ & $8.1 \pm 0.6$ \\
\cline { 2 - 4 } & Eq. S1 & $24 \pm 2$ & $2.6 \pm 0.8$ \\
\hline \multirow{2}{*}{$\mathbf{1}^{\mathbf{P M e P h} 2}$} & Eq. S2 & $34 \pm 1$ & $9.0 \pm 0.8$ \\
\cline { 2 - 4 } & Eq. S1 & $21 \pm 2$ & $2.8 \pm 2.0$ \\
\hline Free $\mathrm{N}_{3}^{-}$ & Eq. S1 & $51 \pm 1$ & $1.5 \pm 0.1$ \\
\hline
\end{tabular}




\section{S5. Estimation of $D_{m}^{D S E}$ based on the Debye-Stokes-Einstein equation}

According to the Debye-Stokes-Einstein equation, the orientational diffusion coefficient can be determined from the following expression ${ }^{7-9}$ :

$$
D_{m}^{D S E}=\frac{k_{B} T}{\eta V \lambda(\rho)} .
$$

where $k_{B}$ is the Boltzmann constant. T is room temperature $(298 \mathrm{~K}) . \eta$ is the viscosity of the solution, and here we use the viscosity of the THF solvent at $25^{\circ} \mathrm{C}(0.48 \mathrm{cP})$ based on the low concentrations $(\sim 12 \mathrm{mM})$ of copper complex solutions. $\mathrm{V}$ is the molecular volume which can be determined with the DFT calculations. The copper complexes are approximated as spheroids, and $\rho$ is the ratio of the short to long semiaxes of the spheroid. $\lambda(\rho)$ is a dimensionless friction coefficient. The values of $\lambda(\rho)$ under the slip and stick boundary conditions have been tabulated as a function of $\rho^{8-10}$.

The dimensions of copper complex molecules $(\mathrm{a}, \mathrm{b}, \mathrm{c})$ are determined by using the editconf module in the GROMACS software (version 5.1.2) ${ }^{11}$ and the values are tabulated in Table S5 along with the molecular volumes obtained from DFT calculations. The orientational diffusion coefficients calculated under slip boundary condition $\left(D_{m}^{D S E \text {,slip }}\right)$, stick boundary condition $\left(D_{m}^{D S E, \text { stick }}\right)$, and obtained from anisotropy measurements $\left(D_{m}\right)$ are shown in Table S6. Here $\rho$ is determined as the ratio of $\mathrm{c}$ to the average of $\mathrm{a}$ and $\mathrm{b}$, and $\lambda$ is the interpolated value.

Table S5. Dimensions (a, b, c) and molecular volumes (V) of the three copper complexes.

\begin{tabular}{|c|c|c|c|c|}
\hline & $\mathrm{a}(\mathrm{nm})$ & $\mathrm{b}(\mathrm{nm})$ & $\mathrm{c}(\mathrm{nm})$ & $\mathrm{V}\left(\mathrm{nm}^{3}\right)$ \\
\hline $\mathbf{1}^{\text {PMe3 }}$ & 1.044 & 0.997 & 0.722 & 0.594 \\
\hline $\mathbf{1}^{\text {PMe2Ph }}$ & 1.508 & 1.414 & 0.730 & 0.802 \\
\hline $\mathbf{1}^{\text {PMePh2 }}$ & 1.589 & 1.490 & 0.835 & 1.081 \\
\hline
\end{tabular}

Table S6. Parameters for calculating orientational diffusion coefficient from Debye-Stokes-Einstein equation.

\begin{tabular}{|c|c|c|c|c|c|c|}
\hline & $\rho$ & $\lambda^{\text {slip }}(\rho)$ & $\lambda^{\text {stick }}(\rho)$ & $\begin{array}{c}D_{m}^{D S E, \text { slip }} \\
\left(10^{-3} \mathrm{ps}^{-1}\right)\end{array}$ & $\begin{array}{c}D_{m}^{D S E, \text { stick }} \\
\left(10^{-3} \mathrm{ps}^{-1}\right)\end{array}$ & $\begin{array}{c}D_{m}\left(10^{-3}\right. \\
\left.\mathrm{ps}^{-1}\right)\end{array}$ \\
\hline $\mathbf{1}^{\text {PMe3 }}$ & 0.71 & 0.44 & 6.0 & 33 & 2.4 & 8.0 \\
\hline $\mathbf{1}^{\text {PMe2Ph }}$ & 0.50 & 1.77 & 6.8 & 6.0 & 1.6 & 4.5 \\
\hline $\mathbf{1}^{\text {PMePh2 }}$ & 0.54 & 1.45 & 6.8 & 5.5 & 1.2 & 2.8 \\
\hline
\end{tabular}

From the Table S6 we can see that both $D_{m}^{D S E \text {,slip }}$ and $D_{m}^{D S E \text {,stick }}$ decrease with the addition of phenyl groups, which is consistent with the experimentally measured $D_{m}$. The complete orientational relaxation of the bound azide group is closely related to the orientational diffusion of the whole copper complex molecule. Note that from Section S6 of SI we learn that the experimentally measured $D_{m}$ is the upper limit, but all three $D_{m}^{D S E, s l i p}$ values are larger than $D_{m}$. In addition, the molecular sizes of the copper complexes are much larger than that of the solvent THF molecules, which is consistent with the stick boundary condition. Thus, the copper complexes likely undergo orientational diffusion under the stick boundary condition. 


\section{S6. Examining the reliability of $t_{0 r, 2}$ extracted from biexponential fitting}

As stated above, the orientational relaxation of bound azide occurs on a longer time than can be probed given the vibrational lifetime, and this influences the accuracy of the slow component time constant $t_{o r, 2}$. In order to examine the reliability of $t_{o r, 2}$ values, we fix $t_{o r, 2}$ at different values in the biexponential function and plot the fitting residuals which are shown in Figure S16, S17, and S18 for $\mathbf{1}^{\text {PMe3 }}, \mathbf{1}^{\text {PMe2Ph }}, \mathbf{1}^{\text {PMePh2}}$, respectively. The biexponential fitting without restriction of $t_{\mathrm{or}, 2}$ will be referred to as free biexponential fitting in the following text and the residuals are also shown for each molecule.

By comparing the fitting residuals shown in Figure $\mathrm{S} 16, \mathrm{~S} 17$, and $\mathrm{S} 18$, we find that fixing $\mathrm{t}_{\mathrm{or}, 2}$ to longer timescales than those extracted from the unconstrained biexponential fitting does not influence the residuals at later delay times, except for $\mathbf{1}^{\mathbf{P M e} 3}$ where the average residual at later delay time is above zero when $t_{\text {or, } 2}$ is fixed at $100 \mathrm{ps}$. When setting $\mathrm{t}_{\mathrm{or}, 2}$ to timescales shorter than the extracted parameter the residuals at later time delays differ, falling below zero, which is more prominent when $t_{\mathrm{or}, 2}$ is fixed at timescales that lie towards the lower end of the error bars resulting from the unconstrained biexponential fitting. Thus, the $t_{\mathrm{or}, 2}$ value extracted from unconstrained biexponential fitting can be considered as a lower limit. Therefore, the cone semiangles and $\tau_{c}$ values extracted from applying wobbling-in-a-cone analysis on the free biexponential fitting results are also the lower limits of the corresponding parameters.
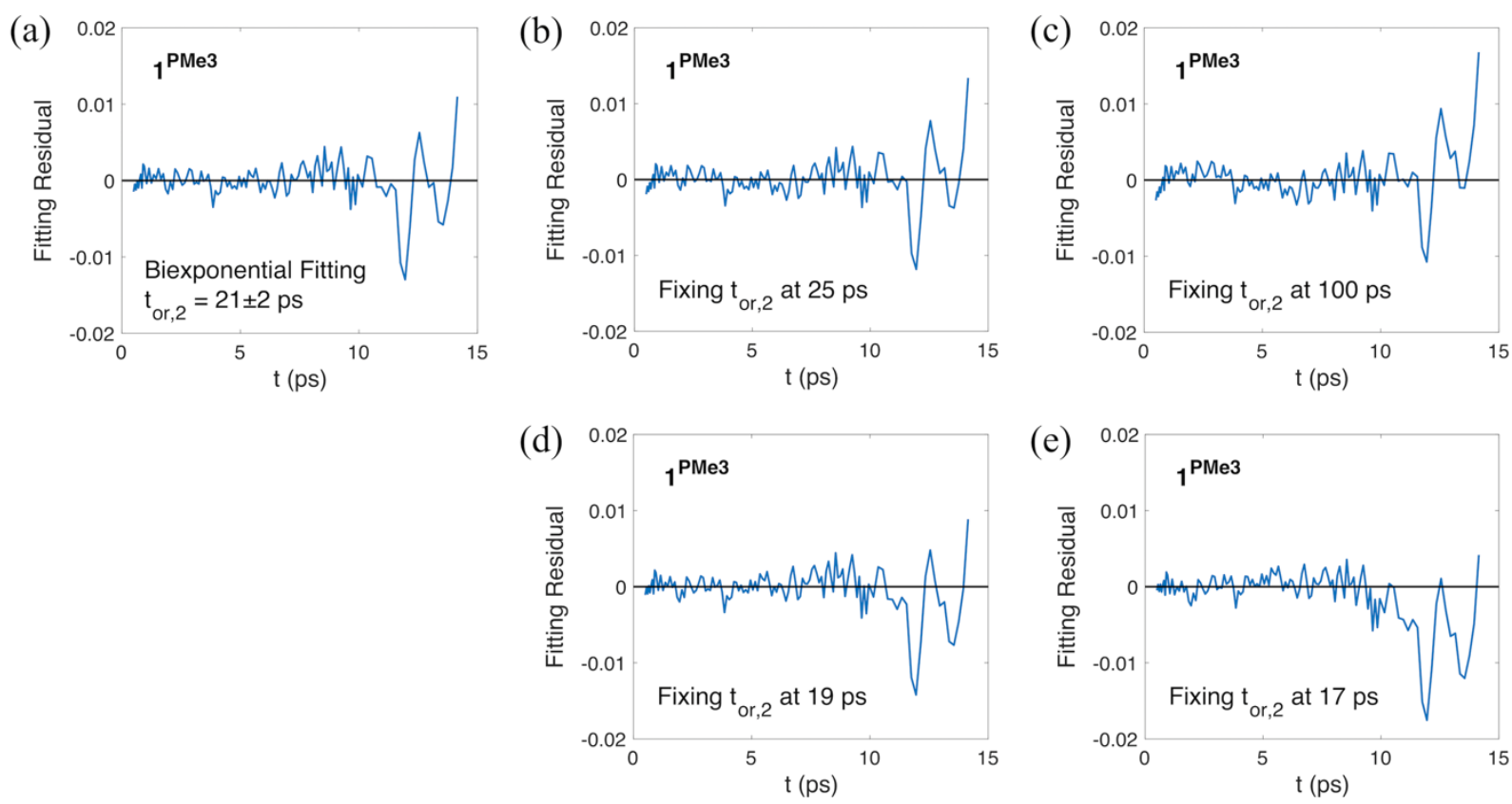

Figure S16. Fitting residuals from the anisotropy decay for $\mathbf{1}^{\mathrm{PMe} 3}$. (a) The residual from the unconstrained biexponential fitting, without restriction of $t_{\mathrm{or}, 2}$, where $\mathrm{tor}, 2_{2}$ was determined to be $21 \pm 2$ ps. (b)-(e) The residuals obtained when tor,2 is fixed to longer timescales ((b) $25 \mathrm{ps}$ and (c) $100 \mathrm{ps)}$ and shorter timescales ((d) $19 \mathrm{ps}$ and (e) 17 ps). 

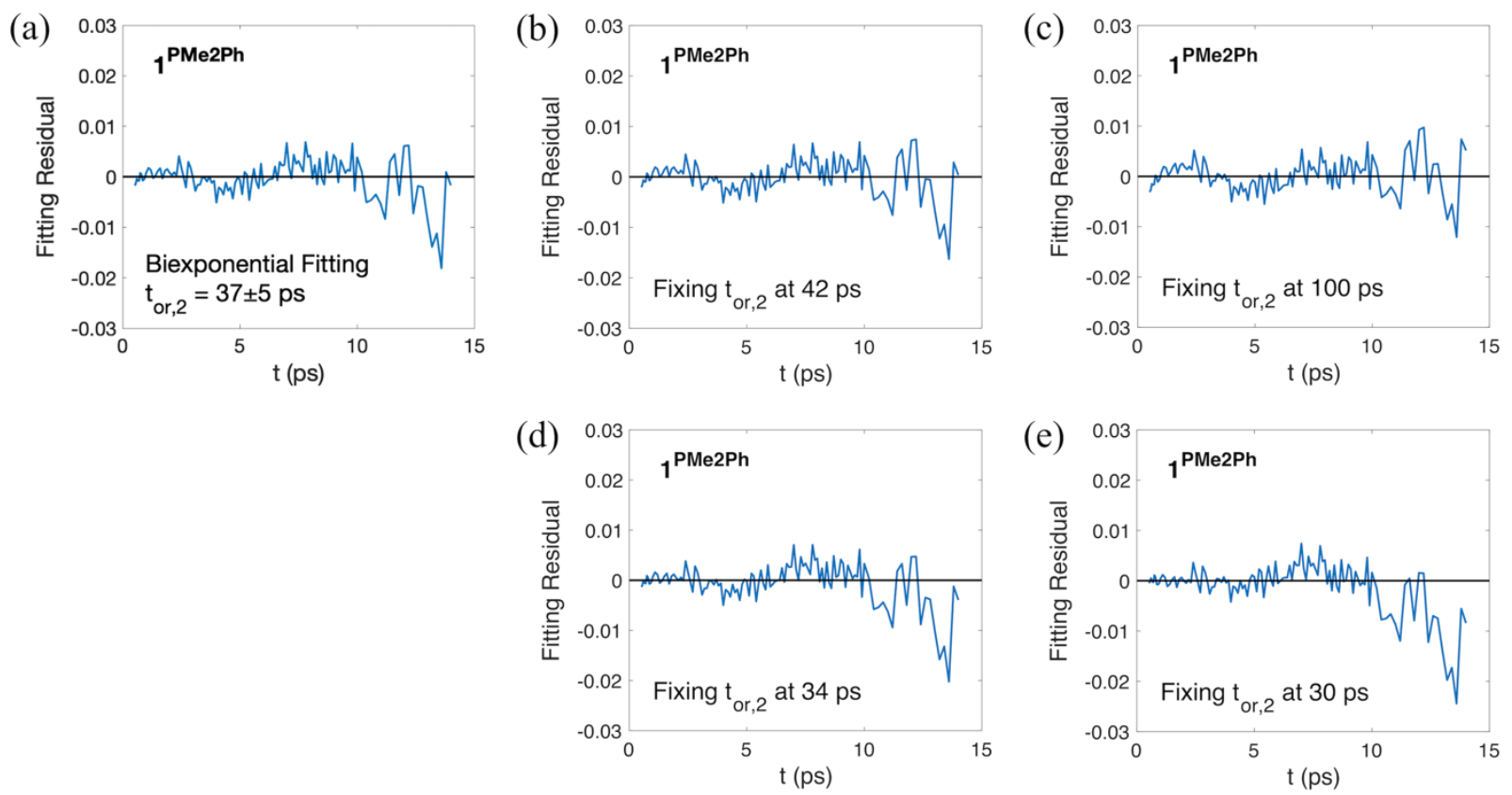

Figure S17. Fitting residuals from the anisotropy decay for $\mathbf{1}^{\mathrm{PMe} 2 \mathrm{Ph}}$. (a) The residual from the unconstrained biexponential fitting, without restriction of $t_{\mathrm{or}, 2}$, where $t_{\mathrm{or}, 2}$ was determined to be $37 \pm 5$ ps. (b)-(e) The residuals obtained when $\mathrm{t}_{\mathrm{or}, 2}$ is fixed to longer timescales ((b) $42 \mathrm{ps}$ and (c) $100 \mathrm{ps)}$ and shorter timescales ((d) $34 \mathrm{ps}$ and (e) 30 ps).
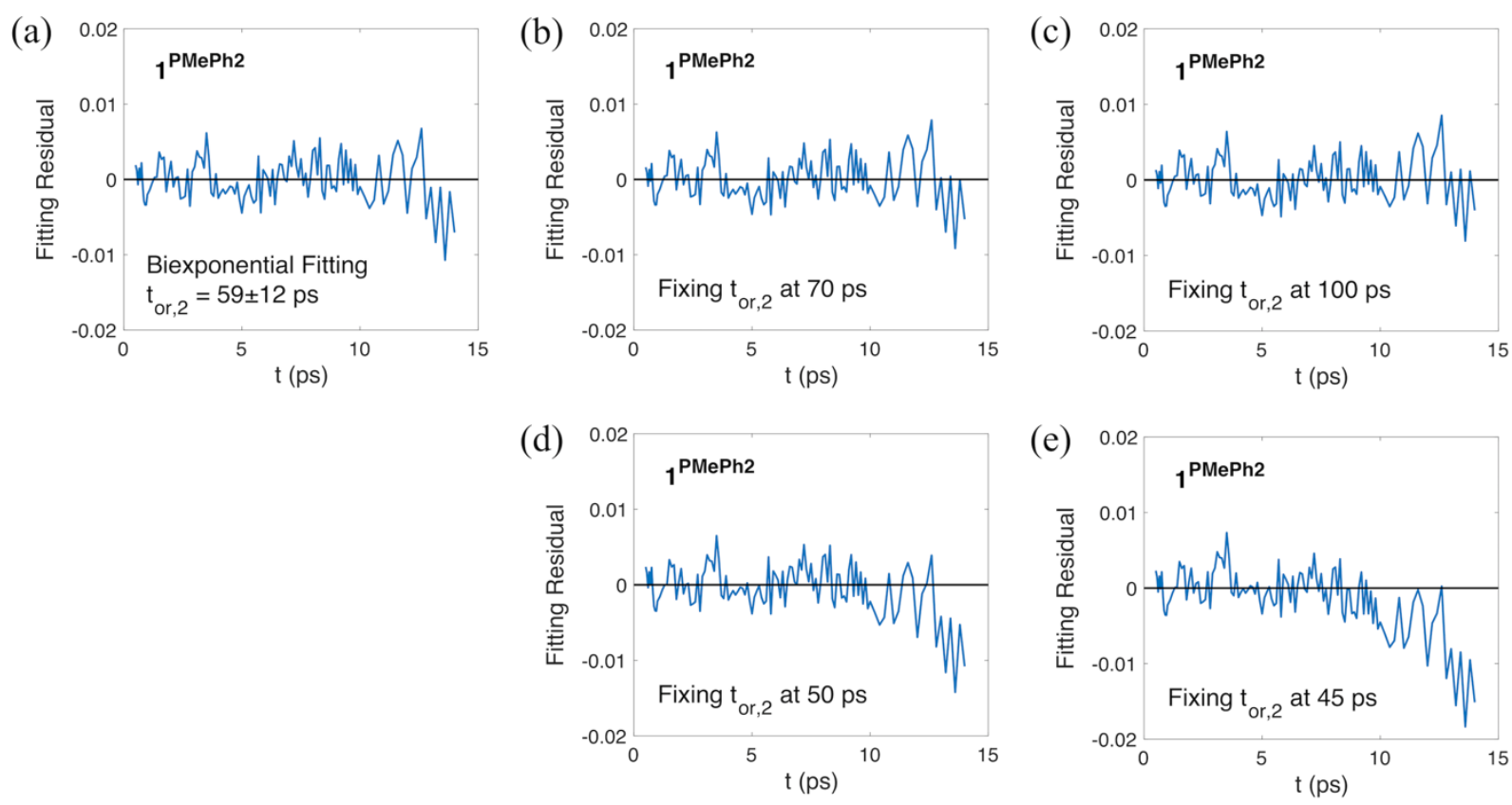

Figure S18. Fitting residuals from the anisotropy decay for $\mathbf{1}^{\mathrm{PMePh} 2}$. (a) The residual from the unconstrained

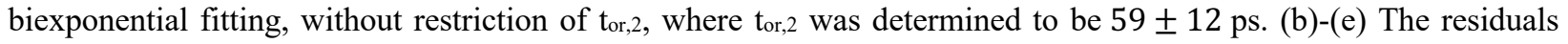
obtained when tor,2 is fixed to longer timescales ((b) $70 \mathrm{ps}$ and (c) $100 \mathrm{ps)}$ and shorter timescales ((d) $50 \mathrm{ps}$ and (e) 45 ps). 


\section{S7. Use of weighting functions when fitting anisotropy decay}

As stated in the main text, the anisotropy decay curves are fit using the corresponding normalized population decay as the fitting weights. Without using the weighting function, large error bars are observed because the decay occurs on a longer timescale such that only a small fraction of the decay curve is captured. The biexponential fitting parameters without the use of weights are shown in Table S7. The fitting parameters of $\mathbf{1}^{\mathrm{PMe} 3}$ are the same within error range when comparing the results with and without the use of weights and the errors are slightly smaller when using the weighting function. As for $\mathbf{1}^{\mathbf{P M e 2 P h}}$ and $\mathbf{1}^{\mathbf{P M e P h} 2}$ the errors increase dramatically without using the weighting function especially for the $t_{2, \text { or }}$. Thus, we choose to use weighting functions when fitting the anisotropy data to account for the decreased signal associated with the population decay.

The choice of the normalized population decay as the weighting function is motivated by the fact that the anisotropy is calculated from the parallel and perpendicular polarization signals as shown in Equation 2 of the main text, and thus stronger signal with higher signal-to-noise ratio will result in less error for the calculated anisotropy. The population time traces describe the isotropic signal intensity decay and thus can be used as an approximation of the reliability of anisotropy data at each time point.

Table S7. Biexponential fitting of the anisotropy decay $r(t)=a_{1} \exp \left(-\frac{t}{t_{1, o r}}\right)+a_{2} \exp \left(-\frac{t}{t_{2, o r}}\right)$ without using the corresponding normalized population decay as weights. The errors in this table represent $95 \%$ confidence interval.

\begin{tabular}{|c|c|c|c|c|}
\hline & $\mathbf{a}_{\mathbf{1}}$ & $\mathbf{t}_{\mathbf{1}, \mathbf{o r}}(\mathbf{p s})$ & $\mathbf{a}_{\mathbf{2}}$ & $\mathbf{t}_{\mathbf{2 , o r}}(\mathbf{p s})$ \\
\hline $\mathbf{1}^{\text {PMe3 }}$ & $0.07 \pm 0.03$ & $3.6 \pm 1.3$ & $0.27 \pm 0.03$ & $22 \pm 3$ \\
\hline $\mathbf{1}^{\text {PMe2Ph }}$ & $0.14 \pm 0.09$ & $6.9 \pm 3.4$ & $0.21 \pm 0.08$ & $263 \pm 1383$ \\
\hline $\mathbf{1}^{\text {PMePh2 }}$ & $0.07 \pm 0.13$ & $8.2 \pm 10.6$ & $0.27 \pm 0.13$ & $130 \pm 333$ \\
\hline
\end{tabular}




\section{S8. Detailed procedure to extract FFCF parameters}

The frequency frequency correlation function (FFCF) is expressed as ${ }^{12,13}$ :

$$
\mathrm{FFCF}(\mathrm{t})=\frac{\delta(\mathrm{t})}{\mathrm{T}_{2}}+\Delta_{1}^{2} \exp \left(-\frac{\mathrm{t}}{\tau_{1}}\right)+\Delta_{2}^{2} \exp \left(-\frac{\mathrm{t}}{\tau_{2}}\right)
$$

where two inhomogeneous contributions are included for the azide group studied in this work. In order to obtain the parameters $\mathrm{T}_{2}, \Delta_{1}, \tau_{1}, \Delta_{2}, \tau_{2}$, the CLS curves extracted from 2DIR spectra and the experimental FTIR spectra are fit according to the method reported by the Fayer group ${ }^{14}$. The CLS curves are fit with biexponential functions: $\operatorname{CLS}(\mathrm{t})=\mathrm{A}_{1} \exp \left(-\frac{\mathrm{t}}{\mathrm{t}_{1, \mathrm{CLS}}}\right)+\mathrm{A}_{2} \exp \left(-\frac{\mathrm{t}}{\mathrm{t}_{2, \mathrm{CLS}}}\right)$. Since CLS represents the normalized FFCF, $\tau_{1}$ and $\tau_{2}$ are equal to $t_{1, C L S}$ and $t_{2, C L S}$, respectively. $\Delta_{2}$ is calculated as:

$$
\Delta_{2}=\sqrt{\frac{A_{2}}{A_{1}+A_{2}}} \times \frac{F W H M}{2 \sqrt{2 \ln 2}}
$$

where FWHM is the full width at half maximum of the linear FTIR spectrum. $\mathrm{T}_{2}$ and $\Delta_{1}$ are obtained by fitting experimental FTIR spectra with the linear absorption simulated using the response function. To fit the linear absorption spectrum we use the Kubo lineshape function (obtained from integrating Eq.S4 twice) ${ }^{13}$ :

$$
g(t)=\frac{t}{T_{2}}+\Delta_{1}^{2} \tau_{1}^{2}\left(e^{-\frac{t}{\tau_{1}}}+\frac{t}{\tau_{1}}-1\right)+\Delta_{2}^{2} \tau_{2}^{2}\left(e^{-\frac{t}{\tau_{2}}}+\frac{t}{\tau_{2}}-1\right)
$$

Eq.S6 is then used to describe the lineshape function, $g(t)$, in the linear response function:

$$
R^{(1)}(t) \propto e^{i \omega_{01} t-g(t)}
$$

where $\omega_{01}$ is the transition frequency of azide. Taking the real part of the Fourier transform of $\mathrm{R}^{(1)}(\mathrm{t})$ yields the simulated absorption spectrum:

$$
A(\omega) \propto \Re \int_{0}^{\infty} R^{(1)}(t) e^{-i \omega t} d t
$$

Three parameters in Eq. S6 are varied in the fitting process: $\mathrm{T}_{2}, \Delta_{1}$, and $\omega_{01}$. Note that $\tau_{1}$ and $\tau_{2}$ are taken from the fit of the CLS curves and $\Delta_{2}$ is obtained as described in Eq. S5. The fitting results for the FTIR spectra are shown in Figure S19.

Note that the experimental spectral line shape is asymmetric and the degree of asymmetry increases as the number of phenyl groups increases. This might be due to the fact that the orientation of the copper-bound azide group has a spatial distribution, and the interactions with peripheral phosphine ligands vary with different orientations, resulting in a change in both the absorption frequency and transition dipole moment, which causes the asymmetric lineshape in the absorption spectrum. However, the linear response function is constructed with cumulant expansion truncated after second order, leading to a symmetric distribution of vibrational frequencies. Thus discrepancy exists between the experimental and simulated FTIR spectra. 

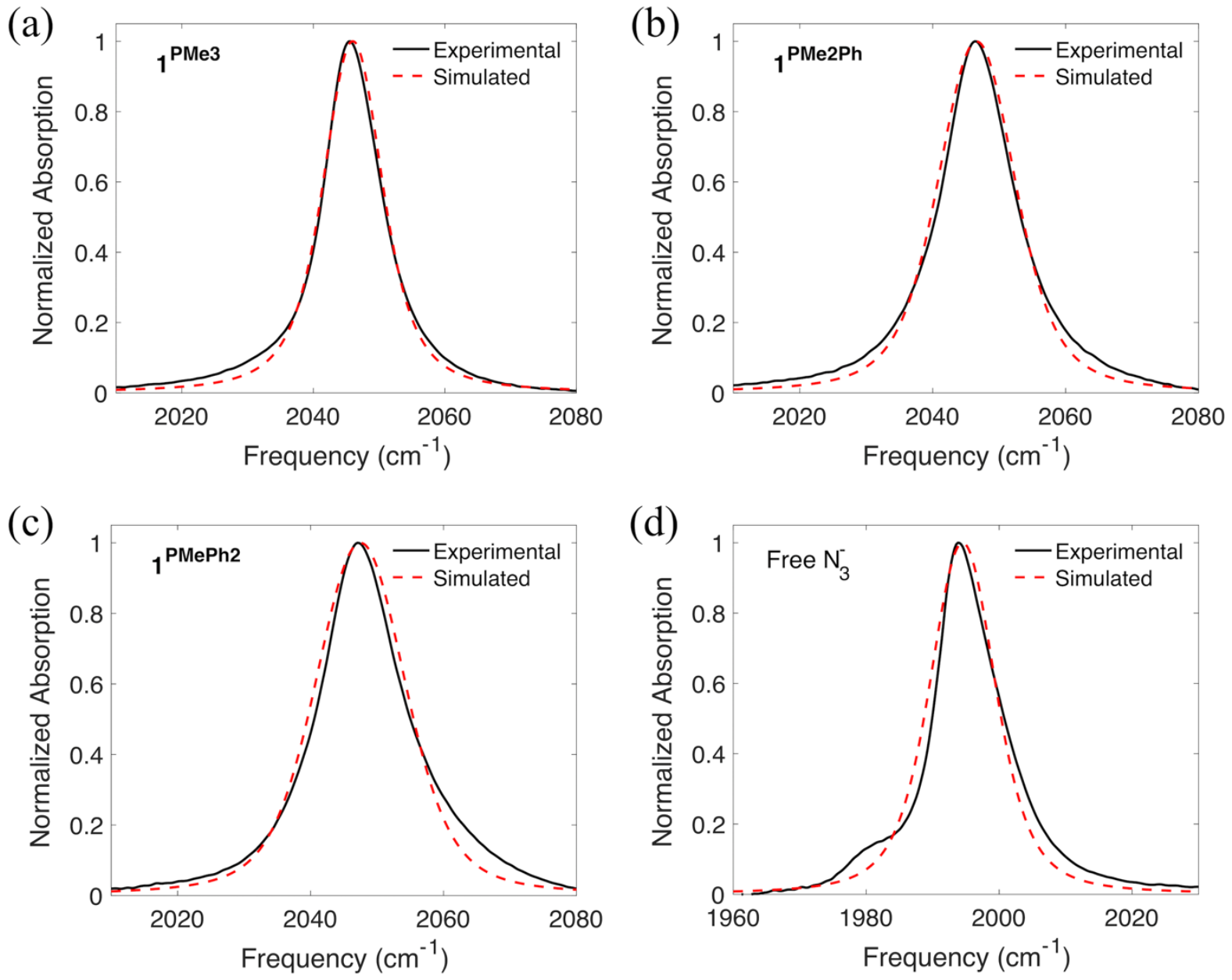

Figure S19. Comparison of experimental FTIR spectra (black solid line) and simulated spectra (red dashed line) for (a) $\mathbf{1}^{\text {PMe3 }}$, (b) $\mathbf{1}^{\text {PMe2Ph }}$, (c) $\mathbf{1}^{\text {PMePh2 }}$, and (d) free $\mathrm{N}_{3}^{-}$. 


\section{S9. Details of error propagation}

The widely adopted methods for error propagation and the calculation of curve fitting errors are described in this section.

When the parameter to be calculated $\mathrm{x}$ is a function of independent variables $\mathrm{a}, \mathrm{b}, \mathrm{c}$ :

$$
x=f(a, b, c) .
$$

The formula for the error propagation adopted in this study is:

$$
\sigma_{x}=\sqrt{\left(\frac{\partial x}{\partial a}\right)^{2} \sigma_{a}^{2}+\left(\frac{\partial x}{\partial b}\right)^{2} \sigma_{b}^{2}+\left(\frac{\partial x}{\partial c}\right)^{2} \sigma_{c}^{2}}
$$

where $\sigma_{a}, \sigma_{b}$, and $\sigma_{c}$ are the standard deviation of the corresponding variables.

S9.1 Error propagation for the wobbling-in-a-cone analysis parameters

The formula for calculating the error of $\theta_{\text {lib }}, \theta_{c}, \theta_{\text {tot }}, \tau_{c}, D_{c}, \tau_{m}, D_{m}$ are:

$$
\begin{aligned}
& \sigma \theta_{\text {lib }}=\sqrt{\left(\frac{\partial \theta_{\text {lib }}}{\partial a_{1}}\right)^{2} \sigma \mathrm{a}_{1}^{2}+\left(\frac{\partial \theta_{\text {lib }}}{\partial a_{2}}\right)^{2} \sigma \mathrm{a}_{2}^{2}} ; \\
& \sigma \theta_{\mathrm{c}}=\sqrt{\left(\frac{\partial \theta_{\mathrm{c}}}{\partial a_{1}}\right)^{2} \sigma \mathrm{a}_{1}^{2}+\left(\frac{\partial \theta_{\mathrm{c}}}{\partial a_{2}}\right)^{2} \sigma \mathrm{a}_{2}^{2}} ; \\
& \sigma \theta_{\mathrm{tot}}=\frac{\partial \theta_{\mathrm{tot}}}{\partial a_{2}} \sigma a_{2} ; \\
& \sigma \tau_{\mathrm{c}}=\sqrt{\left(\frac{\partial \tau_{\mathrm{c}}}{\partial \mathrm{t}_{1, \mathrm{or}}}\right)^{2} \sigma \mathrm{t}_{1, \mathrm{or}}^{2}+\left(\frac{\partial \tau_{\mathrm{c}}}{\partial \mathrm{t}_{2, \mathrm{or}}}\right)^{2} \sigma \mathrm{t}_{2, \mathrm{or}}^{2}} ; \\
& \sigma \mathrm{D}_{c}=\sqrt{\left(\frac{\partial \mathrm{D}_{\mathrm{c}}}{\partial \theta_{c}}\right)^{2} \sigma \theta_{c}^{2}+\left(\frac{\partial \mathrm{D}_{\mathrm{c}}}{\partial \tau_{\mathrm{c}}}\right)^{2} \sigma \tau_{c}^{2}} \\
& \sigma \tau_{m}=\sigma \mathrm{t}_{2, o r}\left(\tau_{\mathrm{m}} \text { is equal to } \mathrm{t}_{2, o r}\right) \text {; } \\
& \sigma \mathrm{D}_{m}=\frac{\partial \mathrm{D}_{m}}{\partial \tau_{\mathrm{m}}} \sigma \tau_{m}
\end{aligned}
$$

S9.2 Error propagation for the FFCF parameters

$\tau_{1}$ and $\tau_{2}$ are equal to $t_{1, \text { CLS }}$ and $t_{2, \text { CLS }}$ respectively, therefore the error of $\tau_{1}$ and $\tau_{2}$ are equal to those of $\mathrm{t}_{1, \mathrm{CLS}}$ and $\mathrm{t}_{2, \mathrm{CLS}}$ :

The error of $\Delta_{2}$ can be calculated as:

$$
\begin{aligned}
& \sigma \tau_{1}=\sigma \mathrm{t}_{1, \mathrm{CLS}} ; \\
& \sigma \tau_{2}=\sigma \mathrm{t}_{2, \mathrm{CLS}} .
\end{aligned}
$$




$$
\sigma \Delta_{2}=\sqrt{\left(\frac{\partial \Delta_{2}}{\partial \mathrm{A}_{1}}\right)^{2} \sigma \mathrm{A}_{1}^{2}+\left(\frac{\partial \Delta_{2}}{\partial \mathrm{A}_{2}}\right)^{2} \sigma \mathrm{A}_{2}^{2}}
$$

$\mathrm{T}_{2}$ and $\Delta_{1}$ are obtained by fitting the experimental FTIR spectrum $\mathrm{A}_{\mathrm{E}}\left(\mathrm{w}_{\mathrm{i}}\right)$ with the simulated FTIR spectrum $A_{S}\left(w_{i}\right)$, where $\mathrm{w}_{i}(i=1,2,3, \ldots, N)$ are the points on the frequency axis, and $A_{E}\left(w_{i}\right)$ and $\mathrm{A}_{\mathrm{S}}\left(\mathrm{w}_{\mathrm{i}}\right)$ are the absorption at each frequency for the experimental and simulated spectra respectively. The function fminsearchbnd in Matlab is used to obtain optimized $\mathrm{T}_{2}$ and $\Delta_{1}$ parameters by minimizing the following expression:

$$
\mathrm{S}=\sum_{\mathrm{i}=1}^{\mathrm{N}}\left[\mathrm{A}_{\mathrm{E}}\left(\mathrm{w}_{\mathrm{i}}\right)-\mathrm{A}_{\mathrm{S}}\left(\mathrm{w}_{\mathrm{i}}\right)\right]^{2}
$$

The minimized value of $\mathrm{S}$ will be denoted as $\mathrm{S}_{0}$. Then we calculate the variance of the residuals:

$$
\text { Sigma }=\frac{\mathrm{S}_{0}}{\mathrm{~N}-\mathrm{k}}
$$

where $\mathrm{N}$ is the number of data points, and $\mathrm{k}$ is the number of fitting parameters, in this case, $3\left(\mathrm{~T}_{2}\right.$, $\Delta_{1}$, and center frequency of the FTIR spectrum, $\left.\omega_{01}\right)$. The function jacobianest in Matlab is used to estimate the Jacobian matrix, J, of the simulated FTIR spectrum with respect to the three fitting parameters at the optimized values. The QR decomposition of J by using the $q r$ function in Matlab yields an upper triangular matrix R:

$$
[\mathrm{Q}, \mathrm{R}]=\operatorname{qr}(\mathrm{J}, 0)
$$

with which we can calculate the covariance matrix:

$$
\operatorname{Cov}=\operatorname{Sigma} \times\left(\mathrm{R}^{-1} *\left(\mathrm{R}^{-1}\right)^{\mathrm{T}}\right)
$$

where superscript -1 stands for the inverse of a matrix and superscript $T$ stands for the transpose of a matrix. The vector containing the error of the three fitting parameters ( $95 \%$ confidence interval) is:

$$
\left(\begin{array}{c}
\sigma \mathrm{T}_{2} \\
\sigma \Delta_{1} \\
\sigma \omega_{01}
\end{array}\right)=2 \times \sqrt{\operatorname{diag}(\mathrm{Cov})}
$$

where the factor 2 is an estimate of the $97.5 \%$ quantile of Student's t distribution with a large degree of freedom (N-k), and diag is a Matlab function used to extract the diagonal elements of the Cov matrix.

The error of $\boldsymbol{\Gamma}_{2}$ is:

$$
\sigma \Gamma_{2}=\frac{\partial \Gamma_{2}}{\partial T_{2}} \sigma T_{2}
$$




\section{S10. References}

(1) Weberg, A. B.; McCollom, S. P.; Thierer, L. M.; Gau, M. R.; Carroll, P. J.; Tomson, N. C. Using Internal Electrostatic Fields to Manipulate the Valence Manifolds of Copper Complexes. Chem. Sci. 2021, No. 12, 4395-4404.

(2) APEX3 2016.1-0: Bruker-AXS, Madison, Wisconsin, USA (2016).

(3) SAINT v8.38A: Bruker-AXS, Madison, Wisconsin, USA (2014).

(4) Krause, L.; Herbst-Irmer, R.; Sheldrick, G. M.; Stalke, D. Comparison of Silver and Molybdenum Microfocus X-Ray Sources for Single-Crystal Structure Determination. $J$. Appl. Crystallogr. 2015, 48, 3-10.

(5) Sheldrick, G. M. SHELXT - Integrated Space-Group and Crystal-Structure Determination. Acta Crystallogr. Sect. A Found. Crystallogr. 2015, 71, 3-8.

(6) Sheldrick, G. M. Crystal Structure Refinement with SHELXL. Acta Crystallogr. Sect. A 2015, 71, 3-8.

(7) Yamada, S. A.; Bailey, H. E.; Tamimi, A.; Li, C.; Fayer, M. D. Dynamics in a RoomTemperature Ionic Liquid from the Cation Perspective: 2D IR Vibrational Echo Spectroscopy. J. Am. Chem. Soc. 2017, 139, 2408-2420.

(8) Youngren, G. K.; Acrivos, A. Rotational Friction Coefficients for Ellipsoids and Chemical Molecules with the Slip Boundary Condition. J. Chem. Phys. 1975, 63, 3846-3848.

(9) Sension, R. J.; Hochstrasser, R. M. Comment on: Rotational Friction Coefficients for Ellipsoids and Chemical Molecules with Slip Boundary Conditions. J. Chem. Phys. 1993, 98, 2490.

(10) Hu, C.-M.; Zwanzig, R. Rotational Friction Coefficients for Spheroids with the Slipping Boundary Condition. J. Chem. Phys. 1974, 60, 4354-4357.

(11) Abraham, M. J.; Murtola, T.; Schulz, R.; Páll, S.; Smith, J. C.; Hess, B.; Lindah, E. GROMACS: High Performance Molecular Simulations through Multi-Level Parallelism from Laptops to Supercomputers. SoftwareX 2015, 1-2, 19-25.

(12) Kubo, R. A Stochastic Theory of Line Shape. Adv. Chem. Phys. 1969, 15, 101-127.

(13) Hamm, P.; Zanni, M. T. Concepts and Methods of 2D Infrared Spectroscopy; Cambridge University Press: New York, 2011.

(14) Kwak, K.; Park, S.; Finkelstein, I. J.; Fayer, M. D. Frequency-Frequency Correlation Functions and Apodization in Two-Dimensional Infrared Vibrational Echo Spectroscopy: A New Approach. J. Chem. Phys. 2007, 127, 124503. 